Franz Lebsanft, Angela Schrott

\title{
Diskurse, Texte, Traditionen
}

\section{Ausgangspunkt und Ziel}

Grundlage und Rahmen des vorliegenden, aus einer Sektion des Berliner Romanistentags hervorgegangenen Bandes ist die in den letzten Jahren intensiv erforschte und kontrovers diskutierte Funktion der Diskurstraditionen, die als kulturelles und sprachbezogenes Wissen die Gestaltung von Texten anleiten und der Angelpunkt für eine kulturbezogene sprachwissenschaftliche Textanalyse sind. In der Romanistik hat in den vergangenen Jahren die Diskussion um die Relation der Diskurstraditionen zu den anderen in Texte und Diskurse eingehenden Regeln und Traditionen breiten Raum eingenommen. Die rahmensetzende Bezugsgröße für die meisten Beiträge war (und ist) dabei das von Eugenio Coseriu in den 1950er Jahren erstmals konzipierte System der kulturellen Sprachkompetenz mit der Trias seiner auf den drei Ebenen des Universellen, des Historischen und des Individuellen betrachteten Seinsweise der Sprache als energeia, dynamis und ergon (Coseriu 1955-1956, 1988). Diese Bezugnahme auf das Coseriu'sche Modell hat es der Romanistik ermöglicht, die Historizität und Kulturalität von Sprache über die Analyse einzelner Phänomene hinaus stets rückgebunden an ein linguistisches System zu untersuchen.

Ein fundamentales Charakteristikum der aktuellen romanistischen Forschung ist daher, dass die meisten, von Brigitte Schlieben-Langes Traditionen des Sprechens (1983) angestoßenen und von Peter Koch (1997) und Wulf Oesterreicher (1997) entscheidend vorangebrachten Diskussionen zu dem inzwischen vorwiegend nur noch „Diskurstraditionen“ genannten Phänomenbereich sich innerhalb des Coseriu'schen Paradigmas bewegen. Der in allen Kulturwissenschaften präsente Begriff des "Diskurses" ist jedoch selbst in dem engeren Bereich der Linguistik polysem. Parallel zu dem aufgerufenen und in der deutschsprachigen Romanistik dominanten Paradigma haben sich in Frankreich und Deutschland - hier vor allem in der Germanistik - ganz andere Spielarten der linguistischen Diskursanalyse (analyse du discours) und inzwischen auch eine veritable Diskurslinguistik etabliert, die mit durchaus „entfernt“ ver- 
wandten, vor allem von Michel Foucault $(1969,1971)$ ausgehenden Diskursbegriffen arbeiten, aber gleichwohl andere Methoden und Perspektiven privilegieren. Auch diese Forschungsrichtung ist in diesem Band vertreten. Ein wichtiges - angesichts der nicht zu nivellierenden Unterschiedlichkeit der Paradigmen zweifellos nur teilweise eingelöstes - Ziel der Sektionsarbeit war es daher, die Diskussion um die Diskurstraditionen stärker als bisher mit Modellen und Methoden zu verknüpfen, die in analoger, doch in andere Richtungen zielender Weise mit der Historizität und Kulturalität von Texten befasst sind. Ein zweiter, in mehreren Beiträgen erkennbarer interdisziplinärer Aspekt der Sektionsarbeit war innerhalb des romanistischen Paradigmas die Herstellung um nicht zu sagen: Wiederbelebung - des Gesprächs zwischen Philologie, Sprach- und Literaturwissenschaft, das vom Gesichtspunkt der Diskurstraditionenforschung umso vielversprechender ist, als diese in den Anfängen, bei Coseriu (1980/31994), ohnehin in der Sprache der Dichtung die Seinsweise der Sprache in ihrer Vollkommenheit verwirklicht sieht und daher vorwiegend an literarischen Beispielen demonstriert wird. Eine weitere Konvergenz der im Band versammelten Beiträge ist eine verstärkte empirische Fundierung der Diskurstraditionenforschung. Nach einer Phase intensiver theoretischer Diskussionen zeigt sich aktuell eine Tendenz zu korpusbasierten Studien. Diese Betonung der Empirie impliziert, dass die individuelle Ebene der Diskurse und Texte im Rahmen des Coseriu'schen Modells und seiner Rezeption eine Revalorisierung erfährt. Diese zunehmend empirische Ausrichtung wird unseres Erachtens dazu beitragen, einen immer noch weitgehend „weißen Fleck“ der Diskurstraditionenforschung zu kartographieren, und zwar die kulturellen Gruppierungen, die in den einzelnen Sprachgemeinschaften oder sprachübergreifend das kulturelle Wissen der Diskurstraditionen ausüben, variieren und in verwandelter Form weitergeben.

Wir beabsichtigen nicht, die einzelnen Beiträge dieses Bandes, die für sich selbst sprechen, näher zu präsentieren oder zu kommentieren. Eine globale Einordnung und Orientierung, aus der die Anlage des Bandes hervorgeht, mag genügen. Die Beiträge umfassen im ersten Kapitel theoretische, zum Teil kontrovers geführte Diskussionen im Rahmen des von Coseriu gesetzten Paradigmas, mit gelegentlichen Öffnungen hin zur germanistischen Forschung. Im zweiten Kapitel finden sich linguistische Fallstudien, in denen sich die methodische Vielfalt von Diskurstraditionenforschung und Diskurslinguistik deutlicher manifestiert. Der dritte Teil behandelt schließlich philologische und literaturwissenschaftliche - theoretische wie praktische - Aspekte einer Auseinandersetzung und Verarbeitung linguistischer Theoriebildung. Was wir mit den folgenden Überlegungen hingegen versuchen wollen, ist die hoffentlich nicht nur romanistischen Leserinnen und Leser einerseits an den Diskussionsstand der linguistischen Diskurs- und Diskurstraditionenforschung innerhalb unseres 
Fachs behutsam heranzuführen, andererseits die Punkte zu markieren, an denen die divergenten Optionen der (germanistischen) Diskursanalyse und Diskurslinguistik zumindest im Ansatz deutlich werden. Ein gangbarer Weg, um dieses Ziel zu erreichen, scheint uns eine Darstellung und Diskussion der Kernbegriffe, welche die Basis der in diesem Band aufgerufenen Forschungsrichtungen bilden.

\section{Diskurs und Text: Zur Konstituierung zweier Gegenstände der Linguistik}

Wer die Traditionen der Diskurse und Texte zum Gegenstand der linguistischen Analyse macht, setzt die Verwendung von Begrifflichkeiten voraus, deren Verständnis alles andere als selbstverständlich ist. Denn obwohl Diskurs und Text seit geraumer Zeit linguistisch terminologisiert sind, bietet vor allem der erstere der beiden Begriffe ganz erheblichen Interpretationsspielraum, mit einschneidenden Konsequenzen für Forschungsschwerpunkte und -perspektiven. Daher konzentrieren wir unsere Überlegungen im Folgenden auf den dank seiner philosophischen Nobilitierung leuchtenden (und möglicherweise auch blendenden) Leitbegriff des Diskurses und führen in dessen Schatten denjenigen des Textes ohne vergleichbare Problematisierung nur mit.

In der ersten Auflage des bekannten und gut orientierenden Lexikons der Sprachwissenschaft (Bußmann 1983: 103f.) firmiert Diskurs zunächst im Sinne von Jürgen Habermas als

Verständigung über problematisierte Geltungsansprüche von Meinungen und Normen, die bei dem kommunikativen Handeln, dem eingelebten und normativ abgesicherten Austausch handlungsbezogener Erfahrungen, naiv vorausgesetzt werden.

Erst an zweiter Stelle und dabei nur äußerst knapp wird Diskurs auch als „Folge von Äußerungsakten “ im Sinne der Konversationsanalyse definiert (Bußmann 1983: 104). In der zweiten Auflage des Lexikons dreht sich die Reihenfolge und Gewichtung der Definitionen um, denn nunmehr wird Diskurs an erster Stelle behandelt als „aus der angloamerikan[ischen] Forschung übernommener Oberbegriff für verschiedene Aspekte von Text", zu denen die Interpretation des Begriffs als „zusammenhängende Rede“, als „geäußerter Text“, als „kohärenter Text“, als „vom Sprecher für einen Hörer konstruierter Text“ und als „Ergebnis eines interaktiven Prozesses im soziokulturellen Kontext“ gezählt werden (Bußmann ${ }^{2}$ 1990: 189). Erst nach dieser linguistischen Bestimmung wird nunmehr auf den Habermas'schen "philosophischen Kontext" verwiesen; hingegen ist von Michel Foucaults damals längst schon prominentem Diskursbegriff, der heute in der Gesamtheit der Kulturwissenschaften zweifellos 
dominiert, überhaupt noch keine Rede. In der dritten Auflage des Lexikons wird als ein weiterer Aspekt der ersten Definition „Diskurs als ,Prozess' im Unterschied zu Text als ,Produkt“" genannt (Bußmann ${ }^{3}$ 2002: 171; vgl. ${ }^{4} 2008$ : 141). Gewichtiger noch ist, nach den wiederum knappen Ausführungen zum Habermas'schen Diskursbegriff, die Aufnahme einer dritten, an Foucault anknüpfenden Definition. Demnach bezeichne Diskurs

eine Menge von inhaltlich zusammengehörenden Texten oder Äußerungen, die nicht wie im Diskurs (1) [d. h. Diskurs als Text] - in einer realen Gesprächssituation verknüpft sind, sondern ein intertextuelles, Gespräch' in einer Kommunikationsgemeinschaft bilden. (Bußmann ${ }^{3} 2002$ : 171; vgl. Bußmann ${ }^{4} 2008: 141$ )

Die Grundlagen linguistischer Diskurskonzepte wurden bereits in den 1950er Jahren entwickelt und beruhen - was z.B. bei Warnke (2007) nicht deutlich wird - auf der Diskussion einer die moderne Linguistik immer noch fundierenden Dichotomie Ferdinand de Saussures. In Opposition zur langue definiert Saussure (1916/2013: 80) die parole bekanntlich als einen „acte individuel de volonté et d'intelligence“, in dem sich die "combinaisons par lesquelles le sujet parlant utilise le code de la langue en vue d'exprimer sa pensée personnelle“ feststellen ließen. Um dieses aus seiner Sicht nicht nur individuelle, sondern als solches auch akzessorische Objekt im Deutschen zu benennen, schlägt Saussure den Ausdruck Rede vor, der allerdings den Nachteil habe, parole eine besondere Bedeutung hinzuzufügen, und zwar die von (französisch) discours im Sinne einer umfassenderen Äußerung, die über den "acte individuel“ hinausgeht. Zwar betont Saussure ausdrücklich, dass es eine schlechte Methode sei, von den Wörtern auszugehen, um Gegenstände zu definieren; doch die Wahl von parole - und eben nicht von discours - zur Bezeichnung des „acte individuel de volonté et d'intelligence" scheint seiner Charakterisierung der konkreten Sprechtätigkeit besonders Rechnung zu tragen. Es ist daher höchst aufschlussreich, dass der von Saussure offenbar gerade vermiedene Ausdruck discours - bzw. seine formale Entsprechung in anderen Sprachen - genau dort in der Diskussion auftaucht, wo es im Widerspruch zu den Auffassungen des Genfer Linguisten um die Frage möglicher systematischer Aspekte der parole geht. In der allgemeinen Sprachwissenschaft gilt dies bereits für Zellig S. Harris' (1952) Skizze einer distributionell und formal vorgehenden und auf diese Weise systematische Textstrukturen herausarbeitenden discourse analysis ebenso wie für Eugenio Coserius (1955-1956) nur wenig jüngeren Entwurf einer funktionellen lingüistica del hablar.

Harris (1952: 1) definiert discourse als „connected speech (or writing)“, dessen formale Analyse er anhand eines (schriftlich vermittelten) Textes - es handelt sich um einen Werbetext - exemplifiziert, um so Einsichten über „the structure of a text or a type of text" (1952: 30) zu gewinnen. Zwar privilegiert 
Harris das Problem einer Ausdehnung der deskriptiven Linguistik auf sprachliche Äußerungen „beyond the limits of a single sentence at a time“, d. h. also die Entwicklung einer transphrastischen Grammatik, die für ihn eine Theorie der Texttypen offenbar einschließt; doch formuliert er zugleich ein zweites, in Nordamerika später von der Ethnomethodologie und der Ethnographie des Sprechens behandeltes Problem, das in der Verknüpfung von Kultur und Sprache bestehe (1952: 1). Im Hinblick auf den letzteren Aspekt stellt Harris (1952: 3) einfach fest, dass jeder "connected discourse" stets eingebettet sei in eine außersprachliche Situation, „whether of a person speaking, or of a conversation, or of someone sitting down occasionally over a period of months to write a particular kind of book in a particular literary or scientific tradition". Während in Frankreich Harris' (1952) discourse analysis programmatisch in einem entsprechenden, von Jean Dubois und Joseph Sumpf herausgegebenen Themenheft der Zeitschrift Langages als analyse du discours (Harris 1969) übersetzt wurde, wählte man bei der Übersetzung ins Deutsche das nicht weniger programmatische Konzept der Textanalyse (Harris 1976), so dass man wenige Jahre später diesen Beitrag als „Gründungsurkunde der heute [d. h. also damals] so lebhaft betriebenen Textlinguistik“ (Szemerényi 1982: 38) feiern konnte. Noch Warnke (2007: 4) charakterisiert Harris’ Begriff von discours im Rahmen der Textlinguistik:

Unter discours versteht Harris in etwa das, was später in der Germanistischen Linguistik als Text bezeichnet wird, also eine satzüberschreitende sprachliche Äußerungsform, das, was man auch als transphrastische Einheit bezeichnet.

Was Coseriu (1955-1956: 31) betrifft, so plädiert er in seiner das sogenannte ,Drei-Ebenen-Modell' der Sprache entwickelnden "Linguistik des Sprechens“ dafür, den Begriff der parole durch denjenigen des hablar (Sprechen) zu ersetzen, und dieses hablar - offenbar in der Nachfolge von Antonino Pagliaro (1955) - als individuelle „Tätigkeit" discurso, als individuelles „Produkt“ texto zu nennen. In der Tat interpretiert Pagliaro (1955: 5) die parole als

il momento soggettivo della lingua, l'atteggiamento particolare che la funzionalità del sistema assume nell'atto in cui si attua come discorso.

Unglücklicherweise hat der deutsche Übersetzer (Uwe Petersen, Coseriu 1975: 257) den Ausdruck discurso ausgerechnet durch Rede wiedergegeben und damit gerade die von Saussure sich distanzierenden begrifflichen Distinktionen Coserius verwischt, wie die Gegenüberstellung des spanischen Originals (1955-1956: 31) mit der deutschen Übersetzung (1975: 257) zeigt: 


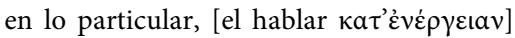
es el discurso (el acto o la serie de actos) de tal individuo en tal oportunidad;

En lo particular, el habla como "producto“ es, justamente, el texto; im besonderen ist es die Rede (Sprechakt oder Reihe von Sprechakten) eines bestimmten Einzelnen zu der oder der Gelegenheit;

Im besonderen ist das Sprechen als „Produkt" eben der Text;

Mag man Harris zu Gute halten, dass er die Einbettung des Sprechens in eine nicht-sprachliche Situation immerhin problematisiert, so ist die Erfassung und Beschreibung dieser Einpassung für Coseriu zentral, weil für ihn das Sprechen im Unterschied zur Sprache nicht „a-circunstancial“ sei (1955-1956: 34). Er löst - wie ebenso bekannt ist - den analytischen Zugriff auf die Situation durch eine höchst komplexe Ausdifferenzierung des fundamentalen Bühler'schen Konzepts des Umfelds (Coseriu 1955-1956: 46), die Brigitte Schlieben-Lange später gerade dort aufgreifen wird, wo sie die „Traditionen des Sprechens“ prominent macht (Schlieben-Lange 1983: 13-25).

Die Einführung des Ausdrucks discours in die französischsprachige Linguistik verdankt sich vermutlich einer lange vor dem schon erwähnten Themenheft von Langages einsetzenden, kritischen Auseinandersetzung mit Harris. In der Tat konstruiert Émile Benveniste (1954/1976: 11) eine zunächst noch nicht näher erläuterte Opposition langue vs. discours in Anlehnung an Harris (1951), dem er vorwirft, beides miteinander gleichzusetzen. In Benveniste (1956a/1976: 251) erscheint dann der discours als individuelle Aktualisierung der langue in der parole, und zwar dort, wo er von der übereinzelsprachlichen Natur der Pronomina spricht, die sich in den

„instances de discours“, c'est-à-dire les actes discrets et chaque fois uniques par lesquels

la langue est actualisée en parole par un locuteur,

manifestiere. Zur selben Zeit bietet die Beschäftigung mit Freuds Traumdeutung Benveniste (1956b/1976: 78) die Gelegenheit, die verschiedenen discours der Sprecher als je einmalige „configurations de la parole“ $\mathrm{zu}$ beschreiben, in denen sich ihre Persönlichkeit ausdrücke und konstruiere. Es ist der durch die Sprache vermittelte Zusammenhang zwischen Psychoanalyse und Linguistik, der zu der - so Foucault (1969: 39) - für die Geistesgeschichte (histoire de la pensée) wichtigen Annahme einer dem Sprecher unmittelbar nicht verfügbaren Doppelung der discours führe (Benveniste 1956b/1976: 75f.):

L'analyste opère sur ce que le sujet lui dit. Il le considère dans les discours que celui-ci lui tient, il l'examine dans son comportement locutoire, „fabulateur“, et à travers ces discours se configure lentement pour lui un autre discours [unsere Hervorhebung] qu'il aura charge d'expliciter, celui du complexe enseveli dans l'inconscient. 
Zweifellos in Anknüpfung an Charles Bally (1932/ $1965: 35)$ bezeichnet Benveniste (1959/1976: 241f.) den Mechanismus, durch den sich der discours in der parole konstituiert, als énonciation:

Il faut entendre discours dans sa plus large extension: toute énonciation supposant un locuteur et un auditeur, et chez le premier l'intention d'influencer l'autre en quelque manière.

Im Anschluss kategorisiert Benveniste (1959/1976: 242) die doch einmaligen "Diskurse“ nach Mustern der Tradierung, die er in einer den literaturwissenschaftlichen Gattungsbegriff weit überschreitenden Auslegung als genres bestimmt:

C'est d'abord la diversité des discours oraux de toute nature et de tout niveau, de la conversation triviale à la harangue la plus ornée. Mais c'est aussi la masse des écrits qui reproduisent des discours oraux ou qui en empruntent le tour et les fins: correspondances, mémoires, théâtre, ouvrages didactiques, bref tous les genres où quelqu'un s'adresse à quelqu'un, s'énonce comme locuteur et organise ce qu'il dit dans la catégorie de la personne.

Das Zusammenspiel von langue, parole, énonciation und discours verdeutlicht dann noch einmal eine späte und berühmt gewordene Arbeit, die in dem von Tzvetan Todorov herausgegebenen Themenheft „L'énonciation“ der Zeitschrift Langages publiziert wurde (Benveniste 1970/1981: 80):

L'énonciation est cette mise en fonctionnement de la langue par un acte individuel d'utilisation. Le discours, dira-t-on, qui est produit chaque fois qu'on parle, cette manifestation de l'énonciation, n'est-ce pas simplement la "parole“? - Il faut prendre garde à la condition spécifique de l'énonciation: c'est l'acte même de produire un énoncé et non le texte de l'énoncé qui est notre objet. Cet acte est le fait du locuteur qui mobilise la langue pour son compte.

Man dürfte kaum fehl in der Annahme gehen, dass solche Bestimmungen die zunächst reichlich vage und der eigenen Begriffsbestimmung vorausgehende „utilisation sauvage“ der Ausdrücke énoncé und discours der Archéologie du savoir (Foucault 1969: 44) beeinflussen, die sich freilich über die präzisen Quellen ihrer der zeitgenössischen Linguistik entlehnten Gebrauchsweisen ebenso notorisch ausschweigt wie sie einer genaueren Auseinandersetzung mit ihnen bewusst ausweicht. Beim Versuch einer definitorischen Festlegung von énoncé und discours als theoretisch gesicherte und analytisch brauchbare Begriffe bezeichnet Foucault (1969: 140) die Möglichkeit des Äußerungsakts als performance verbale bzw. performance linguistique, den individuellen Äußerungsakt als formulation, dessen sprachliche Grundeinheit als (grammatische) phrase oder (logische) proposition. Deren Seinsweise (modalité d'existence) werde ermöglicht durch das énoncé, das Äußerungsakte in einem Gegenstandsfeld (domaine d'objets) und im Hinblick auf mögliche Äußerungssubjekte situierte. 
Der discours bildet sich nach Foucault aus sprachlichen Einheiten, insoweit sie énoncés in dem beschriebenen Sinne sind; ihre Grundlage nennt er die formation discursive:

Et si je parviens à montrer [...] que la loi d'une pareille série [d. h. von Zeichen], c'est précisément ce que j'ai appelé jusqu'ici une formation discursive [...], le terme de discours pourra être fixé: ensemble des énoncés qui relèvent d'un même système de formation; et c'est ainsi que je pourrai parler du discours clinique, du discours économique, du discours de l'histoire naturelle, du discours psychiatrique (Foucault 1969: 141).

Diese Definition ist das Ergebnis einer vierfachen und stets als unzulänglich qualifizierten Annäherung an den Begriff der formation discursive, der sich aus den ausführlich entfalteten Bestimmungen des énoncé durch dessen objets (Gegenstände), den style seiner énonciation (Äußerungsmodalitäten), seine concepts (Begriffe) und schließlich seine thèmes (Strategien) speist (Foucault 1969: 45-50). Die strikt abgelehnte Fokussierung der einen oder anderen Bestimmung führt freilich zu einer schillernden Verwendung von discours (1969: 106), wie sie sich in den Beispielen eines „discours de la psychopathologie“ $(1969: 45,55)$, eines „discours de la folie“ (1969: 46), eines - wie bereits im obigen Zitat benannten - „discours cliniqu“ (1969: 47, 72) oder eines "discours économiqu““ (1969: 91) niederschlägt und bereits im Ansatz einer alle Kulturwissenschaften erfassenden Inflationierung Vorschub leistet, die man kaum - wie Fohrmann (1997: 370) anhand der Beispiele juristischer, feministischer, literarischer Diskurs meint - auf exklusiv linguistische Verwendungsweisen zurückführen kann.

Natürlich kann Foucault nicht umhin, als dokumentarische Grundlage seiner großen frühen Werke (besonders Foucault 1966), welche durch die Archéologie du savoir (und noch durch den Ordre du discours, Foucault 1971) retrospektive eine theoretische und methodologische Rechtfertigung erhalten sollen, sprachliche Äußerungen qua performance linguistique zu wählen; doch bilden diese gerade nicht den Gegenstand seiner Untersuchung:

On voit en particulier que l'analyse des énoncés ne prétend pas être une description totale, exhaustive du «langage» ou de «ce qui a été dit». Dans toute l'épaisseur impliquée par les performances verbales, elle se situe à un niveau particulier qui doit être dégagé des autres, caractérisé par rapport à eux, et abstrait. En particulier, elle ne prend pas la place d'une analyse logique des propositions, d'une analyse grammaticale des phrases, d'une analyse psychologique ou contextuelle des formulations: elle constitue une autre manière d'attaquer les performances verbales, d'en dissocier la complexité, d'isoler les termes qui s'y entrecroisent et de repérer les diverses régularités auxquelles elles obéissent (Foucault 1969: 142).

Der Foucault'sche discours lässt sich nach dem Willen seines Schöpfers auf ein nach seinem Verständnis rein sprachlich bestimmtes und linguistisch zu analysierendes Phänomen trotz mancher Berührungspunkte nicht reduzieren. Der 
„ganz andere“ discours der Linguisten (Foucault 1969: 141) bilde vielmehr „l'étage terminal“ des von ihm selbst gemeinten Gegenstands, und zwar als

les textes (ou les paroles) tels qu'ils se donnent avec leur vocabulaire, leur syntaxe, leur structure logique ou leur organisation rhétorique.

Kommt dem linguistischen Gegenstand die „analyse structurale“ (Foucault 1969: 25, 27) bei - das geradezu legendär gewordene Heft 8 (1966) von Communications mit Roland Barthes' (1966) programmatischer, auf Harris und Benveniste Bezug nehmender analyse structurale des récits war drei Jahre vor der Archéologie du savoir erschienen -, so will Foucault die „analyse des formes discursives" gerade davon abheben:

L'analyse [der „formations discursives"] reste en deça de ce niveau manifeste, qui est celui de la construction achevée: en définissant le principe de distribution des objets dans un discours, elle ne rend pas compte de toutes leurs connexions, de leur structure fine, ni de leurs subdivisions internes: en cherchant la loi de dispersion des concepts, elle ne rend pas compte de tous les processus d'élaboration, ni de toutes les chaînes déductives dans lesquelles ils peuvent figurer; si elle étudie les modalités d'énonciation, elle ne met en question ni le style ni l'enchaînement des phrases; bref, elle laisse en pointillé la mise en place finale du texte (Foucault 1969: 100).

Die begrifflichen und methodisch operationalisierbaren Festlegungen, die Foucault dezidiert scheut, leisten die Exegeten, wenn sie den zwischen den „Dingen“ und den „Wörtern“ (Foucault 1966) angesiedelten poststrukturalistischen Diskurs als „apersonales, transindividuelles ,régime`, das gesellschaftliche Wissenssysteme herstellt, in spezifischen ,Formationen' (z.B. Psychologie, Medizin, Sprachwissenschaft) ordnet und aufrecht erhält" definieren (Fohrmann 1997: 370) oder einer "heuristisch-pragmatische[n] Bestimmung“ zuführen, wonach Diskurse „materiell nachweisbare Formen gesellschaftlicher Rede [sind], die stets nach Praxisbereichen spezialisiert und institutionalisiert sind, sodass es Diskurse mit distinkten Formations- und Ausschließungsregeln und jeweils eigener Operativität gibt“" (Parr 2008: 235).

\section{Traditionen der Diskurse und Texte}

Es mag deutlich geworden sein, dass Diskurs und Text Gegenstandkonstruktionen sind, die Linguisten der parole abgewinnen, weil sie Saussure verborgen gebliebene oder von ihm ausgeblendete Phänomene systematischer Strukturiertheit sowie der Muster- bzw. Regelhaftigkeit im, Äußerungsfluss` entdecken. Die störende Akt-Objekt-Ambiguität, die dem Ausdruck Äußerung innewohnt, kann dabei durch die Unterscheidung von ,Enunziation' (énonciation) als Tätigkeit und ,Enunziat‘ (énoncé) als Ergebnis oder Produkt beseitigt werden. 
Ganz gleich, ob man Diskurs und Text synonymisch oder synonymendifferenzierend - im Verhältnis von ,Akt' zu ,Objekt' oder auch von Hyperonym zu Hyponym - verwendet, stets geht es darum, das Individuum überschreitende soziale und kulturelle Bedingungen und Bedingtheiten des je einmaligen Sprechens aufzuspüren und zu beschreiben. Dort, wo man mit Diskurs eher Phänomene der Enunziation, mit Text vorwiegend solche des Enunziats etikettiert, liegt es nahe, linguistische Diskursanalyse als Beschreibung „realer Kommunikation" - vorwiegend als mündlich konzipierten Dialog und Gespräch bzw. Konversation (Ehlich 1994: 10) -, Textanalyse oder Textlinguistik als Beschreibung vor allem schriftlich konzipierter, monologischer Sprachprodukte zu verstehen. Auf diese Weise belegt man freilich zunächst nur zwei Felder einer intuitiv angewendeten Kreuzklassifikation unter Verwendung der Kriterien ,konzeptionelle Versprachlichungsstrategie (Koch/Oesterreicher ${ }^{2} 2011$ ) und ,ontischer Status' des sprachlichen Geschehens (Akt-Objekt-Unterscheidung; vgl. Coseriu 1955-1956):

\begin{tabular}{|c|c|c|}
\hline$\underbrace{\text { Akt-Objekt }}_{\text {Konzeption }}$ & Enunziation & Enunziat \\
\hline mündlich & Diskurs & \\
\hline schriftlich & & Text \\
\hline
\end{tabular}

Kreuzklassifikation I: Diskurs und Text

Die Begrifflichkeit dieser Kreuzklassifikation kann man auf zwei Weisen auf die anderen beiden Felder ausdehnen. Die erste Redeweise, die wir bevorzugen, besteht darin dass man von mündlichen bzw. schriftlichen Diskursen spricht, die zu mündlichen oder schriftlichen Texten im Sinne von Textprodukten ,gerinnen' (Ia): 


\begin{tabular}{|c|c|c|}
\hline${ }_{\text {Konzeption }}^{\text {Akt-Objekt }}$ & Enunziation & Enunziat \\
\hline mündlich & Diskurs & Text \\
\hline schriftlich & $\downarrow$ Diskurs & Text \\
\hline
\end{tabular}

Kreuzklassifikation Ia: Diskurs und Text

Die zweite Möglichkeit ist, dass man von mündlichen Diskursen und schriftlichen Texten spricht, die jeweils als Tätigkeit oder als Produkte in Erscheinung treten $(\mathrm{Ib})$ :

\begin{tabular}{|c|c|c|}
\hline Konzeption & Enunziation & Enunziat \\
\hline mündlich & Diskurs & Diskurs \\
\hline schriftlich & Text & Text \\
\hline
\end{tabular}

Kreuzklassifikation Ib: Diskurs und Text

Die Vielfalt entsprechend unterschiedlich akzentuierender Herangehensweisen an Diskurse bzw. Texte und an Methoden ihrer Erforschung dokumentieren eindrucksvoll die beiden HSK-Bände zur Text- und Gesprächslinguistik von Brinker u. a. (2000-2001).

Der Gedanke, dass die Strukturiertheit satzüberschreitender Äußerungen sei es als Diskurs, sei es als Text - sich der Verwendung von Mustern verdankt, der Sprecher bei ihrer Verfertigung entsprechend spezifischen, vorgefundenen Regeln folgt und diese gegebenenfalls überschreitet und dazu beiträgt, sie zu verändern, ist in der Textlinguistik und in der linguistischen Diskursanalyse von Beginn an systematisch ausgebaut worden. Entsprechend wird in der Textlinguistik - in engem Austausch mit der literaturwissenschaftlichen Gattungstheorie (vgl. schon Hempfer 1973) - mit Konzepten wie Textfunktion, Texttyp, Textmuster oder Textsorte, in der linguistischen Diskurs- als Gesprächsanalyse mit soweit möglich analogen Begriffen wie Gesprächstyp, Gesprächsmuster oder Gesprächssorte gearbeitet. Dies ist der Zusammenhang, in den der von Coseriu in seiner Textlinguistik (1980/31994) vorgeschlagene Terminus der den Textbegriff entscheidend dynamisierenden Texttradition gehört, der von Schlieben-Lange (1983) übernommen und von Peter Koch (1997) sowie Wulf Oesterreicher (1997) unter dem Namen Diskurstradition weiterentwickelt wur- 
de. Bei Coseriu wird der Begriff der Texttradition parallel und differenzierend zum Begriff der Einzelsprache eingeführt, die er als eine „historisch gewordene Tradition des Sprechens" (1980/31994: 6) bezeichnet. Die zuvor getroffene Unterscheidung zwischen einer universellen Betrachtungsebene des Sprechens oder der Sprache „im allgemeinen“ und der Ebene der historischen Einzelsprachen wird also ergänzt um

Die Ebene der Texte, der Redeakte bzw. der Gefüge von Redeakten, die von einem bestimmten Sprecher in einer bestimmten Situation realisiert werden, was natürlich in mündlicher oder in schriftlicher Form geschehen kann. (Coseriu 1980/31994: 7 bzw. 10)

Der Sprecher verfüge entsprechend über eine Tradition, die nicht nur eine Fähigkeit ist, diese oder jene (Einzel)sprache zu sprechen, sondern eben auch (mündliche oder schriftliche) Texte zu gestalten:

Und weiterhin gibt es - auf der individuellen Ebene - eine Fähigkeit, ganz bestimmte Texte zu verfertigen, die ebenfalls [wie die universelle Sprachkompetenz] nichts mit Sprachkenntnissen im herkömmlichen Sinn zu tun hat. Textsorten wie z.B. „Liebesbrief" oder „wissenschaftliche Abhandlung" haben ihre eigene Tradition, die nicht mit einer bestimmten historischen Tradition des Sprechens [d. h. einer Einzelsprache] zusammenfällt. (Coseriu 1980/31994: 28 bzw. 38)

In Fortführung dieser Überlegungen zu,den Textsorten und ihren Traditionen fixiert Coseriu dann den Terminus der Texttraditionen, die einer historischen Sprachtradition - wie z.B. eine bestimmte Grußformel - oder mehreren - wie z. B. literarischen Gattungen - „einverleibt“ werden könnten (vgl. Coseriu 1980/31994: 40 bzw. 53, mit einer verdeutlichenden Formulierung).

Die Annahme einer spezifischen, dynamischen Textkompetenz beruht für Coseriu (1980/31994: 39f. bzw. 52f.) noch auf weiteren Fakten, zu denen er, wie bereits in Coseriu (1955-1956) deutlich wird, die Einbettung des Sprechens und damit der geäußerten Texte - in sozial und kulturell geformte außersprachlichen Kontexte (die bereits erwähnten „Umfelder“) sowie die „Redeuniversen“, d. h. das jeweilige "universelle System von Bedeutungen, zu dem ein Text gehört und durch das er seine Gültigkeit und seinen besonderen Sinn erhält" (Coseriu 1980/31994: 95 bzw. 128), zählt. Als Redeuniversen nennt Coseriu (1980/31994: 100 bzw. 134) beispielhaft „die Mythologie, die Literatur, die Wissenschaft, die Mathematik, unsere praktische Lebenswelt".

Schlieben-Langes Verdienst ist es, diese im theoretischen Kern vollständig übernommene (und begrifflich kaum erneuerte, nämlich nur im Hinblick auf die nunmehr „Diskursuniversen“ genannten „Redeuniversen“ veränderte) Grundkonzeption gewissermaßen „alltagstauglich“ gemacht zu haben, indem sie unter Einbeziehung der soziolinguistischen Formen der Diskursanalyse nordamerikanischer Prägung die Funktion von Texten pragmatisch als Beitrag 
zur Bewältigung oder Entlastung vom Alltag definiert. Es sei die Funktion der Texte, „bestimmte praktische Aufgaben des Zusammenlebens zu lösen oder aber sich in bestimmter Weise für eine Weile diesen praktischen Aufgaben zu entziehen" (Schlieben-Lange 1983: 26). Stärker als Coseriu verbindet sie folglich Textfunktionen und, in Anknüpfung an Erving Goffman (1971), gesellschaftliche Organisationsformen, wobei sie den neuen Gedanken entwickelt, dass Texttraditionen von "soziale[n] oder kulturelle[n] Gemeinschaften“ getragen würden (Schlieben-Lange 1983: 28). Wie man an den Beispielen bei Coseriu (1980) sieht, soll das Konzept der Text- und später dann der Diskurstradition einen Phänomenbereich von den textuellen Mikroformen wie der Grußformel bis hin zu Makroformen wie dem Roman abdecken. Auch Schlieben-Lange (1983: 139f.) schlägt vor, „einfache“ bis „komplexe“ Texttraditionen, von den elementaren sprachlichen Handlungen bis zu den Texttypen, zu erfassen; darüber hinaus perspektiviert sie die Analyse der Texttypen im Hinblick auf die „einer höheren Abstraktionsebene“ angehörenden Diskursuniversen.

Die weitere, von Koch (1997) und Oesterreicher (1997) wesentlich angestoBene Theoriediskussion zu den Diskurs- und Texttraditionen findet in einer Fülle von Sammelbänden (Jacob/Kabatek 2001, Aschenberg/Wilhelm 2003, Schrott/Völker 2005, Kabatek 2008), Handbuchartikeln (Wilhelm 2001) und auch Einzelbeiträgen ihren reichen Ertrag, an den die in dieses Paradigma sich einschreibenden Beiträge dieses Bandes anknüpfen. Folgende Themenkomplexe stehen dabei im Zentrum der Diskussion: das diffizile und nach wie vor strittige Problem der systematischen Verortung des Wandelprozesses und der diachronischen Dynamik von Diskurs- und Texttraditionen; der nicht weniger kontrovers diskutierte Zusammenhang zwischen den Diskurs- bzw. Texttraditionen einerseits und den Sprachvarietäten bzw. historischen Einzelsprachen andererseits; die Ausdifferenzierung unterschiedlicher Typen von Diskurs- und Texttraditionen nach dem Grad ihrer Komplexität und nach ihrer Zuordnung zu verschiedenen Diskursuniversen; die Faktorisierung der Einbettung in kulturelle und soziale Umfelder; schließlich das wiederum ganz unterschiedlich eingestufte Verhältnis von Diskursen und Texten zu ihren als „Diskursgemeinschaften" apostrophierten Sprechergemeinschaften.

Aus den getroffenen Bezugsetzungen von Diskurs und Text ergeben sich für die Benennung des sprachbezogenen kulturellen Wissens verschiedene Möglichkeiten. So wird der Begriff der Texttradition, wie erwähnt, bereits in der Coseriu'schen Textlinguistik verwendet und hat den Vorteil, dass er den forschungsgeschichtlich bereits stark beanspruchten Diskurs-Begriff meidet. Allerdings sprechen vor dem Hintergrund der Kreuzklassifikation gute Argumente für den Begriff der Diskurstradition. Nutzt man die Begriffe „Diskurs“ und „Text“ zur Unterscheidung der Gesichtspunkte energeia und ergon, dann liegt der Begriff der Diskurstradition nahe. Denn da die Tätigkeit dem Produkt vo- 
rausgeht, ist das kulturelle und sprachbezogene Wissen primär ein Leitfaden für die Tätigkeit des Sprechens in konkreten Kommunikationsituationen und erst sekundär ein Wissen, das aus den Produkten dieser Tätigkeit extrahiert wird. Dieser Bezug auf die energeia und damit auf den Diskurs kann vor der Folie der entworfenen Kreuzklassifikation durch den Begriff der Diskurstradition verdeutlicht werden.

\section{Tradition und doppelte Traditionalität des Sprechens}

\subsection{Zur Tradition der Tradition}

Als Wissenstyp sind die Diskurstraditionen durch Historizität und Traditionalität definiert. Doch während die Historizität diskurstraditionellen Wissens kontrastiv zur Geschichtlichkeit der historischen Einzelsprachen mehrfach Gegenstand romanistischer Studien war, wurde die Frage, ob sich Diskurstraditionen und idiomatische Traditionen auch in ihrer Traditionalität unterscheiden, bisher nicht thematisiert.

Klärende Impulse kann das Konzept der Tradition von der Geschichtswissenschaft empfangen, die Traditionen als Lebensgewohnheiten deutet, die in Wissen und Handlungsweisen der Vergangenheit wurzeln und als Muster aktuellen Handelns fungieren. Damit sind Traditionen eine kulturelle und soziale Ausstattung und bilden Handlungsvorgaben, auf die Menschen zur Entlastung des täglichen Handelns rekurrieren (vgl. Assmann ${ }^{5}$ 1997: 35, Schörken ${ }^{5} 1997: 8$, Rüsen 1983: 64, 66-68, 92). Diese Sicht der Tradition als Handlungsvorgabe akzentuiert im System der Sprachkompetenz den Tätigkeitsbezug von Traditionen und damit deren Anbindung an die energeia. Zugleich bildet die Idee der Tradition als Handlungsvorgabe einen Ansatzpunkt für die (historische) linguistische Pragmatik und deren Verständnis sprachlichen Handelns, da auf diese Weise die Traditionalität von Sprechakten in den Fokus rückt.

Im Kontext linguistischer Studien erscheint es jedoch notwendig, den Begriff der Tradition in Ergänzung zur geschichtswissenschaftlichen Betrachtungsweise auch im Kontext sprachwissenschaftlicher Modelle zu orientieren und auf diese Weise linguistisch anzureichern. Der Begriff der Tradition oder Gewohnheit wird in der Romanistik mehrfach als Kernkonzept für eine kulturorientierte Linguistik aufgefasst. In der romanischen Sprachwissenschaft hat der Traditionsbegriff zwei unterschiedliche Fundierungen: eine semiotische und eine historisch-philologische, die für das Konzept der Diskurstraditionen entscheidend ist und daher im Fokus der folgenden Ausführungen steht. 
Die semiotische Fundierung des linguistischen Traditionsbegriffs etabliert Ferdinand de Saussure in seinem Cours de linguistique générale (1916/2013). Für Saussure ist aufgrund der Konzentration auf die Synchronie der langue die Geschichtlichkeit der Sprache zwar sekundär, doch ist der Begriff der Tradition für seine Zeichentheorie unverzichtbar. So führt Saussure die Arbitrarität des sprachlichen Zeichens auf die Kraft der Tradition zurück (Cours de linguistique générale \$2, Premier principe). Jedes in einer Sprachgemeinschaft funktionierende Ausdrucksmittel beruht nach Saussure auf einer "habitude collective“ bzw. einer "convention“ (Saussure 1916/2013: 172). Die Arbitrarität des sprachlichen Zeichens und seine Festlegung qua Konvention und Tradition bedingen sich gegenseitig (1916/2013: 180):

C'est parce que le signe est arbitraire qu'il ne connaît d'autre loi que celle de la tradition, et c'est parce qu'il se fonde sur la tradition qu'il peut être arbitraire.

Das arbiträre Zeichen folgt der Tradition und diese Tradition erst ermöglicht die Arbitrarität des Zeichens. Der Traditionsbegriff ist damit auch für strukturalistische Ansätze grundlegend. Allerdings wird die Traditionalität hier auf die langue und damit auf das idiomatische Wissen, insbesondere die Theorie des sprachlichen Zeichens, begrenzt. Die kulturelle Traditionalität des Sprechens und der im Sprechen erzeugten Texte wird dagegen in der auf Saussure aufbauenden strukturalistischen Sprachwissenschaft nicht berücksichtigt.

Die zweite, im Kontext der Diskurstraditionenforschung ausschlaggebende Fundierung des Traditionsbegriffs geht auf Ramón Menéndez Pidal zurück. Der bei Coseriu zentrale Begriff der Tradition ist wesentlich vom tradicionalismo Ménendez Pidals beeinflusst. Daher wird im Folgenden das Konzept von Tradition und Traditionalität auf der Basis von Menéndez Pidal und Coseriu präzisiert, um Divergenzen und Konvergenzen beider Traditionalitäten herauszuarbeiten.

\subsection{Sprachwandel und poesía tradicional}

Für Menéndez Pidal sind Traditionen die Grundlage aller menschlichen Gemeinschaften und kulturellen Tätigkeiten. Die Tradition ist der bewegliche Rahmen, in dem sich schöpferische und spontane Kräfte entfalten (1952: 39). Der Zusammenhang von Tradition und Kultur findet sich prägnant in dem Beitrag La lengua de Cervantes en las escuelas. Anlässlich der Frage, ob der Quijote eine geeignete Lektüre für Kinder sei, plädiert Menéndez Pidal dafür, dass Kinder diesen Text in Form einer angeleiteten Lektüre in Auszügen rezipieren sollen (1952: 39): 
La tan decantada espontaneidad inviolable del niño es un mito pedagógico; el niño es un ser cultural; la cultura es tradición y dentro de la tradición lo espontáneo, lo inventivo.

Das Textverstehen ist für Menéndez Pidal eine wesentlich traditionelle Angelegenheit, weshalb ein Leser in die Traditionen des zu rezipierenden Textes eingeführt werden muss. Zentral ist - neben dieser Anmerkung zur Traditionalität des Textverstehens - die Definition der Kultur als Tradition, die den Rahmen für das Schöpferische, Spontane und Erfinderische bildet.

Das Konzept der Tradition und der Traditionalität entwickelt Menéndez Pidal zum einen aus seinen Studien zu Sprachwandel und Sprachgeschichte und zum anderen aus seinen Untersuchungen zur poesía tradicional des romancero in Spanien, wobei vor allem die Überlegungen zu Sprachwandel und sprachlichen Traditionen von Coseriu (1957/1974) aufgenommen wurden. Menéndez Pidals Definition der Tradition als ,trasmisión de conocimientos y prácticas con interés social o colectivo" $\left(1942 /{ }^{9} 1991: 458\right)$ ist weit gehalten; ihre definitorische Schärfe erhält sie durch die Ausführungen zum Sprachwandel und zur Rolle der Tradition in der Dichtung des romancero. Der tradicionalismo pidaliano ist damit in exemplarischer Weise philologisch, ermöglicht vielfältige Brückenschläge zwischen Sprache und Literatur und verbindet sprachwissenschaftliche und literaturwissenschaftliche Überlegungen in einer Kulturgeschichte der Tradition. Dabei steht die Tradition im Spannungsfeld von Individuum und Kollektiv und ist durch das Ineinandergreifen von Tradition und Variation wesenhaft charakterisiert (vgl. Garatea Grau 2005: 60, 69, 71).

Für Menéndez Pidal ist die Sprache diejenige kulturelle Tätigkeit, die am stärksten von Traditionen geprägt ist und in der das Wirken einer Gemeinschaft sich am deutlichsten manifestiert. Die Sprache ist wesenhaft „creación tradicional“ und „creación colectiva“ (1942/ $/{ }^{9} 1991: 458$, 1954: 215), sie ist als "actividad espiritual humana“ ein "hecho social“ (1945: 185). Menéndez Pidal identifiziert damit das idiomatische Wissen klar als traditionelles Wissen und seine Ausführungen zu Sprachwandel und Sprachgeschichte sind immer auch die Geschichte idiomatischer Traditionalität.

Ausgangspunkt für die Analyse der Sprache als Tradition ist die historischdiachrone Sicht auf Prozesse des Sprachwandels. Menéndez Pidal sieht Sprachwandel als sozialen und kulturellen Prozess, der keinen allgemeinen Regeln folgt, sondern vielmehr durch die besonderen historischen Bedingungen und sich überlagernden, komplexen kulturellen Relationen in der Sprachgemeinschaft geformt wird (1926/ ${ }^{8} 1976$ : 544). Obwohl sprachliche Veränderungen immer eine Sprechergemeinschaft betreffen, geht der Sprachwandel auf individuelle und einmalige Akte zurück (1945: 196). Die Individuen sind keine passiven Träger der Tradition, sondern eigenen sich Traditionen an, verändern und aktualisieren sie (vgl. auch Garatea Grau 2005: 72-76). 
Diese individuellen, schöpferischen Akte der Innovation können „tendencias colectivas" (1926/81976: 532) bilden, die dann möglicherweise zu allgemeinen Normen werden und eine sprachliche Tradition verändern können. Die permanente Schöpfung von Innovationen bewirkt eine „convivencia“ miteinander konkurrierender Varianten, zwischen denen die Sprecher je nach Situation und kulturellem Kontext als Optionen wählen können $\left(1926 /{ }^{8} 1976\right.$ : 526, 537). Welche Option von den Sprechern bevorzugt wird und sich durchsetzt, hängt von den jeweiligen historischen Bedingungen ab, so dass jeder Sprachwandel und jede Sprache eine von kulturellen Faktoren geprägte "historia particular" hat (1954: 176). Nach Menéndez Pidal ist die Tradition damit Ursprung aller Veränderungen, sie bedingt und formt sprachliche Strukturen und die Struktur ist das beständig im Wandel begriffene Ergebnis der Tradition: „La tradición precede a la evolución o estructuración, es su punto de arranque, y, por lo tanto, la condiciona" (1954: 187). Da das Sprachsystem aus Traditionen besteht, betrifft die Änderung einer Tradition immer das gesamte System (1954: 184-185). Ausgangspunkt dieser Veränderungen sind dabei stets die Sprecher in ihrem individuellen Sprachhandeln. Da sie die sprachlichen Traditionen in jedem Akt des Sprechens an ihren „estímulo expresivo“ (1945: 196) anpassen, ist die Festigkeit gebende, zugleich Varianten erzeugende Tradition die Existenzform der Sprache: „la lengua está en variedad continua y en permanencia esencial“ (1945: 196).

Die zweite Quelle des tradicionalismo pidaliano sind die spanischen romances. Als epische, einfach gebaute und kurze Texte sind sie für Menéndez Pidal die exemplarische Realisierung einer poesía tradicional, die dadurch definiert ist, dass die einzelnen Texte zwar auf individuelle Schöpfungsakte zurückgehen, jedoch von einer sozialen und kulturellen Gruppe tradiert, vorgetragen und dabei beständig variiert werden (1942/ ${ }^{9} 1991$ : 457). Die Schöpfer der romances agieren zugleich als Individuen und als Mitglieder einer sozialen und kulturellen Gruppe und sind von einem „sentimiento de la colectividad“ getragen (1942/ $/ 91991$ : 455). Ein Text der poesía tradicional ist kein zu einem bestimmten Zeitpunkt von einem Individuum geschaffenes Werk, sondern vielmehr die "lenta labor de una serie de geniales cultivadores de la tradición“" (1942/ ${ }^{9} 1991:$ 426). Diese "geniales cultivadores“ der poetischen Tradition sind vor allem die juglares als Interpreten, die Texte vortragen und dabei immer neue Vaianten erschaffen $\left(1942 /{ }^{9} 1991: 421,449,454\right)$. Die romances oder andere Texte der poesía tradicional, wie etwa die cantares de gesta, existieren daher in einer Vielzahl von Varianten, sie sind unfeste und bewegliche Texte und dieser "estado de fluidez" (1953/1968, Bd. II: 445) zwischen den Varianten macht die Existenzform der poesía tradicional aus. Im Gefüge der poesía tradicional und ihrer Variationen können in der räumlichen Verteilung „áreas compactas" und „áreas dispersas“ unterschieden werden. Während die Traditi- 
on in den „áreas compactas“ stabil und kontinuierlich ist, tritt sie in den „áreas dispersas" diskontinuierlich auf, was durch Faktoren wie Migration bedingt sein kann. Die „áreas compactas" bestehen dabei aus einem Zentrum, das Innovationen erzeugt, und lateralen oder peripheren Regionen, die diese Neuerungen dann aufnehmen und ihrerseits konservativer als das Zentrum sind (1953/1968, Bd. II: 989-990).

Das am Beispiel der poesía tradicional erläuterte Wirken von Traditionen geht von Gattungen der oral poetry aus, bei denen die Überlieferung mit permanenter Variation einhergeht: Tradition und Variation erscheinen als zwei Seiten einer Medaille. Ein romance oder ein cantar de gesta besteht aus einer Vielzahl von Elementen und Traditionen, die in der performance variiert werden. In der Diskursdomäne der Literatur erscheint diese Unfestigkeit ungewöhnlich, weshalb Menéndez Pidals Sichtweise einer auf Tradition und Variation basierenden Dichtung seitens der "escuela individualista“, die literarische Werke als einmalige Schöpfungen eines Autors betrachtete, starken Widerspruch erfuhr (1942/ $\left./^{9} 1991: 442\right)$. Allerdings entspricht die Unfestigkeit der romances vielen Textgattungen und Textsorten außerhalb der Literatur, die weniger durchformt sind und relativ viel Raum für Variationen bieten. Entscheidend ist aber vor allem, dass Menéndez Pidal mit seiner Beschreibung der poesía tradicional wesentliche Charakteristika von Diskurstraditionen erfasst.

Fasst man Textgattungen und Textsorten als historisch verfestigte Kombinationen von Diskurstraditionen auf, dann bestehen literarische Gattungen wie romance und cantar de gesta ebenfalls aus Diskurstraditionen. Damit können Wandel und Variation dieser Texte auch auf die Veränderlichkeit und Varianz diskurstraditionellen Wissens bezogen werden. Aus diesem Grund sind viele Beobachtungen zu den romances nicht allein auf die Gestaltung und Tradierung von Textsorten übertragbar, sondern auch auf den Bereich des diskurstraditionellen Wissens allgemein. Denn Menéndez Pidals Gedanken zur Traditionalität der romances thematisieren die kulturelle Ausformung von Texten und sind damit immer auch allgemeine Überlegungen zur diskurstraditionellen Verfasstheit von Diskursen und Textprodukten. Da die romances (und andere Gattungen der oral poetry) zudem unfeste Texte sind, gelten Menéndez Pidals Ausführungen primär nicht dem Gesichtspunkt des Textes als Produkt (ergon), sondern vor allem dem Gesichtspunkt der energeia als „creación tradicional“ und damit dem Diskurs.

Grundlegend für Tradition und Traditionalität ist die Spannung von individueller Kreativität und Gemeinschaft. Sprachliche Traditionen und die Traditionen der romances als diskurstraditionelles Wissen haben gemeinsam, dass sie als Vermittlung von Wissen und Praktiken und als ,actividad colectiva tradicional" (1926/81976: 532) immer von einer Gruppe oder Gemeinschaft getragen werden. Zugleich aber wird eine Tradition stets von Individuen ausgeübt und 
besteht aus vielen individuellen Schöpfungsakten, die von einem „espíritu de la colectividad“ überwölbt werden (1942/ ${ }^{9} 1991$ : 455): Der einzelne Akteur handelt stets als Individuum und als Mitglied einer Gruppe (vgl. auch 1945: 196). Eine Neuschöpfung oder Innovation kann nur zur Tradition werden, wenn sie von vielen Sprechern übernommen wird. Wird eine solche Innovation von einer ausreichend großen Anzahl von Akteuren übernommen, dann geht sie als Variante in die Tradition ein und verändert sie in ihrer Gesamtheit.

Traditionalität ist daher ein Zusammenspiel individueller Akte von Kreativität in einem „cuerpo social“ und bildet ein emergentes System, bei dem das Ganze mehr ist als die Summe der Teile.

\subsection{Festigkeit, Variation, Sichtbarkeit}

Aus dem tradicionalismo pidaliano können für die Charakterisierung von Traditionalitäten die Kriterien von Festigkeit, Variation und Sichtbarkeit abgeleitet werden.

Bei der Beschreibung von sprachlichen Traditionen und poesía tradicional geht Menéndez Pidal mehrfach auf den Einfluss der Akteure als Träger der Traditionen und auf die Festigkeit von Traditionen ein. Dabei führt er als quantitatives Kriterium die Größe der Gruppe oder Gemeinschaft an, die eine Tradition trägt (1942/ $\left./{ }^{9} 1991: 459\right)$. So ändern sich Traditionen, die von vielen Akteuren ausgeübt werden, verhältnismäßig langsam. Der Einzelne hat relativ wenig Anteil an der Tradition als Ganzes, Neuerungen müssen von vielen übernommen werden, um sich durchzusetzen, und folglich gibt es vergleichsweise wenige Neuerungen, die sich nur langsam durchsetzen. Traditionen, die von einer kleinen Gruppe ausgeübt werden, sind dagegen variantenreicher und ändern sich schneller: Der einzelne Akteur hat mehr Einfluss, Neuerungen einzelner Individuen setzen sich leichter und schneller durch.

Da Sprachgemeinschaften in aller Regel weit mehr Akteure umfassen als eine Diskurstradition, sind idiomatische Traditionen zumeist weniger variantenreich und verändern sich langsamer als diskurstraditionelle Wissensbestände. Diskurstraditionen, insbesondere Traditionen literarischer Gattungen, werden nur von ausgewählten Mitgliedern einer Sprachgemeinschaft beherrscht. In dieser Gruppe, die wesentlich kleiner ist als eine Sprachgemeinschaft, können sich Innovationen leichter durchsetzen, weshalb Diskurstraditionen und insbesondere literarische Traditionen in höherem Maße veränderlich sind als sprachliche Traditionen. Innerhalb der Literatur ist die Traditionalität je nach Gattung unterschiedlich hoch. Je weniger Akteure eine literarische Gattung und deren Traditionen ausüben, umso leichter können sich Varianten durchsetzen und eine Tradition als Ganzes verändern. So sind nach Menéndez Pidal die 
romances in festere Traditionen eingebunden als die cantares de gesta, da sie als kurze Formen von mehr Akteuren praktiziert werden als die längeren cantares, die von der kleineren Gruppe der weitgehend professionellen juglares tradiert werden, in der sich Varianten leichter etablieren.

Hinsichtlich der Festigkeit und Varianz von Traditionen ergibt sich damit für beide Traditionalitäten des Sprechens, dass mit einer größeren Zahl an Akteuren eine höhere Festigkeit einhergeht. Als "actividad social humana“ (Menéndez Pidal 1945: 195) und als traditionelle Tätigkeit unterliegt das Sprechen umso stärker Regelhaftigkeiten, je größer die Zahl der Akteure ist. Da Sprachen überwiegend eine weit größere Basis an Akteuren haben als (insbesondere literarische) Diskurstraditionen, sind einzelsprachliche Traditionen zumeist durch höhere Festigkeit, geringere Varianz und stärkere Regelhaftigkeit charakterisiert.

Eine weitere Differenz zwischen sprachlichen Traditionen und Diskurstraditionen kann aus der Beobachtung Menéndez Pidals abgeleitet werden, dass sprachliche Traditionen die einzige Traditionalität darstellen, die von allen Angehörigen eines „cuerpo social“ beständig ausgeübt wird und damit die Totalität aller Äußerungen einschließt (1926/8 1976: 533). Sprachliche Traditionen werden nicht nur per definitionem - sie konstituieren die Sprachgemeinschaft von allen Mitgliedern dieser Gemeinschaft beherrscht und ausgeübt, sie haben auch die Eigenschaft, dass sie in jeder Äußerung der Mitglieder dieser Gemeinschaft präsent sind, sofern ein Sprecher nicht eine andere Sprache wählt und damit gleichsam zeitweise aus der Sprachgemeinschaft austritt. Betrachtet man dagegen eine kulturelle Gruppierung, die eine bestimmte Diskurstradition - sei es ein wissenschaftlicher Vortrag, eine Predigt oder ein cantar de gesta - beherrscht, dann üben die Mitglieder dieser Gruppe diese Diskurstradition immer nur zeitweise und mit Unterbrechungen aus. Während einzelsprachliche Traditionen damit die Totalität der Äußerungen eines Sprechers als Mitglied der Sprachgemeinschaft prägen, manifestieren sich Diskurstraditionen nur partiell in den Äußerungen der Akteure, die diese Tradition beherrschen.

Eine weitere Unterscheidung Menéndez Pidals, deren Tauglichkeit für die Differenzierung von Diskurstraditionen ebenfalls zu überprüfen wäre, ist der von ihm für die poesía tradicional formulierte Unterschied zwischen kontinuierlichen „áreas compactas" und diskontinuierlichen „áreas dispersas“. Ein mögliches Unterscheidungskriterium von Diskurstraditionen wäre demnach deren kontinuierliche oder diskontinuierliche Tradierung und die Frage, ob bei der Überlieferung eine Ordnung von Zentrum und Peripherie auszumachen ist oder nicht.

Mit dem Kriterium der Festigkeit eng verbunden sind Varianz und Variantenreichtum innerhalb der Traditionalitäten. Menéndez Pidal beschreibt den Zusammenhang von Tradition und Variante sowohl in der Sprache als auch in 
der Dichtung. In der Sprache zeigt sich der Reichtum an Varianten in der Sprachgeschichte vor allem in den literarischen und nicht-literarischen Texten der época preliteraria, deren Sprache noch reicher ist als die aktuellen Dialekte, da es in dieser Epoche noch keine etablierte Sprachnorm gibt (1926/ ${ }^{8} 1976$ : 515, 526). Daher hat der Sprecher - und Schreiber - zahlreiche Optionen und wählt aus dem Inventar der Varianten „según el tono y la ocasión del discurso, según las influencias pasajeras que se entrecruzan en la miente mientras se produce el acto lingüístico." (1926/81976: 526). Aufgrund dieser Vielfalt hat jedes Wort seine eigene Geschichte, sowohl was die historische Semantik als auch was den Lautwandel angeht (1926/81976: 530).

Für die poesía tradicional sind die Varianten die der Gattung gemäße Existenzform (1953/1968, Bd. I: 39). Das Rezitieren ist in unterschiedlichem Grad immer auch eine Neuschöpfung und diese „re-creación colectiva“ erzeugt Textvarianten, mit denen sich ein romance oder ein cantar de gesta kontinuierlich durch Raum und Zeit bewegt (1953/1968, Bd. I: 42). Varianten, die übernommen werden, gewinnen kollektiven Charakter und können eine Tradition verändern. Diese Varianten sind nicht vollkommen frei, sondern bewegen sich im Rahmen von Mustern und besitzen stets zugleich Flexibilität und Stabilität. Die Kreativität der romances ist darin der sprachlichen Kreativität vergleichbar: Jedes Individuum spricht in neuer Weise, bleibt aber innerhalb des Systems seiner Sprache, um verstanden zu werden, und in analoger Weise richtet sich auch der Interpret eines romance nach den Traditionen seiner Gemeinschaft, um rezipiert zu werden (1953/1968, Bd. I: 44). Da sich Varianten in Sprache und poesía tradicional in einem stabilen Rahmen bewegen, zielt ein zentrales Forschungsinteresse darauf, in den Varianten und im Spielraum der Varianz das Gemeinsame und Musterhafte zu erkennen, und zwar sowohl das Musterhafte in den Textprodukten als auch in den Prozessen der Veränderungen, die hinter den verschiedenen Textfassungen stehen (1953/1968, Bd. II: 446-447).

Grundsätzlich können Varianten relativ gleichberechtigt oder aber in ihrer Prominenz deutlich differenziert sein. Dies gilt in der Sprachgeschichte, wenn sprachliche Varianten unterschiedliches Prestige genießen und aus diesem Grund von den Sprechern ,gewählt' oder ,abgewählt' werden oder wenn ein Dialekt sich gegenüber anderen ursprünglich gleichberechtigten Dialekten als Standard durchsetzt. Doch auch Varianten in der poesía tradicional können unterschiedlichen Status haben: So kann eine Variante aufgrund ihrer Qualität besonders oft als Grundlage für die performance gewählt werden und erlangt dann innerhalb der Varianten eines Textes Normcharakter (1953/1968, Bd. II: 393). Übertragen auf idiomatische Traditionen und Diskurstraditionen wird klar, dass sprachliche Varianten und der Zusammenhang von Varietät und Sprachgeschichte in der Sprache intensiv untersucht wurden, während der Zusammenhang von Variante und Diskurstradition bzw. von diskurstraditio- 
neller Varianz und Textsortengeschichte noch nicht thematisiert wurde. Ein Aufgabenfeld der Diskurstraditionenforschung ist es daher, an konkreten Beispielen zu untersuchen, welche Rolle Spielräume der Varianz und Variantenreichtum für die Geschichte von Textsorten und Gattungen spielen (vgl., im Kontext der Debatte um die „New Philology“, für die das Konzept der Varianz zentral ist, Raible 1997: 130-133, 138-140).

Eine weitere von Menéndez Pidal akzentuierte Besonderheit von sprachlichen Traditionen und poesía tradicional ist das Phänomen des „estado latente“. Damit ist auf der Ebene der Sprache gemeint, dass idiomatische Traditionen, die in der Sprachgeschichte zu einem bestimmten Zeitpunkt scheinbar plötzlich auftreten, in Wahrheit bereits lange Zeit davor existieren, aber keinen Eingang in die schriftliche Überlieferung fanden und daher "latent" blieben. Die Bündelung individueller Innovationen zu einem „corriente general“ und die Ausbildung von Tendenzen im Sprachwandel erscheint so gesehen als langwieriger Prozess der „convivencia“ miteinander konkurrierender Formen $\left(1926 /{ }^{8} 1976\right.$ : 537), die sich in der schriftlichen Überlieferung nicht abbildet, sondern allenfalls auf Basis der überlieferten Texte partiell rekonstruiert werden kann (1926/81976: 531, 537).

Dieser „estado latente“ charakterisiert nicht allein die idiomatischen Traditionen, sondern auch die poesía tradicional und ist nach Menénedez Pidal für viele kollektive und traditionelle Tätigkeiten typisch. So wie das Sprechlatein und die frühen romanischen Sprachen einen lang andauernden "estado latente“ haben, da sie nicht schriftlich dokumentiert sind, so ist auch die frühe volkssprachliche Literatur lange Zeit nicht sichtbar: Themen, Formen, Textgestalt und Sprache - spätes Sprechlatein, frühe romanische Sprache - entsprechen nicht den Normen der gelehrten lateinischen Dichtung und werden von den clerici von der schriftlichen Überlieferung ausgeschlossen (1942/ ${ }^{9} 1991:$ 431f.). Die Vorstellung, dass die Dichtung der Ausbildung der romanischen Sprachen nachfolgt, weist Menéndez Pidal zurück, denn für ihn sind die Ausbildung der sprachlichen Traditionen und die Ausformung der kulturellen Traditionen des Dichtens - die Diskurstraditionen der Sprecher des romance - untrennbar in einem sprachlichen und zugleich kulturellen Prozess verbunden. Im Transfer auf die Diskurstraditionen leitet sich die Frage ab, inwiefern Diskurstraditionen als kollektive und kulturelle Größe ebenfalls einen „estado latente“ haben. Diese Fragestellung ist dabei keinesfalls auf die zeitlich entferntere Kulturgeschichte begrenzt, sondern auch für die Entstehung neuer Diskurstraditionen in aktuellen, sich rasch verändernden medialen Kontexten relevant. 


\subsection{Tradition, System, Norm}

Zwei weitere wichtige Ansatzpunkte für das Konzept der Tradition finden sich bei Coseriu: zum einen der Zusammenhang zwischen Tradition und System, zum anderen die Klärung des Traditionsbegriffs vor dem Hintergrund der Differenzierung von System und Norm.

Entscheidend für die Idee der Tradition ist die Geschichtlichkeit und Dynamik der Sprache: Die Sprechtätigkeit als energeia bewirkt, dass die Ausdrucksfreiheit der Sprecher beständig Neues schaffen kann, das zur Gewohnheit wird und in das System der sprachlichen Traditionen integriert wird (Coseriu 1958/1974: 92). Die Kulturalität der Sprache liegt daher in ihrer Traditionalität: Das Sprechen ist eine kulturelle Tätigkeit, weil es etwas Neues hervorbringt, das gelernt und überliefert werden kann (Coseriu 1958/1974: 92 und 1988: 69).

Damit beinhaltet die Sprache zwei Typen von Kulturgeschichten: die Geschichte der sprachlichen Traditionen des Kulturobjekts Sprache und die Geschichte der Diskurstraditionen, die sprachliche Traditionen selegieren und zu Texten formieren, als Kulturgeschichte zweiten Grades. Analog zur primären Historizität der Sprachen, die die Welt durch Zeichen anreichert, und zur sekundären Historizität der Diskurstraditionen, die darauf aufbauend in der Geschichte stehende Diskurse und Texte schafft (vgl. Albrecht 2003: 50, Kabatek in diesem Band), können zwei Typen von Traditionalität postuliert werden: Die primäre Traditionalität des idiomatischen Wissens als sprachliches Material für die Textkonstitution und die darauf aufbauende sekundäre Traditionalität der Diskurstraditionen, die nach kulturellen Normen aus dem sprachlichen Repertoire eine Auswahl trifft und entscheidet, welche sprachlichen Mittel in einen Text als Exemplar einer Gattung oder Textsorte eingehen und welche nicht.

Die kulturelle Geschichtlichkeit beider Traditionen spiegelt sich auch darin, dass Coseriu (1988: 65) alle drei Ebenen des Sprechens und die ihnen zugeordneten Regeln und Traditionen als Komponenten einer kulturellen Sprachkompetenz betrachtet. Diese Sicht der Tradition findet in aktuellen linguistischen und kulturwissenschaftlichen Definitionen ihre Bestätigung, die Kultur als Summe der Traditionen verstehen, durch die sich eine Gemeinschaft charakterisiert und von anderen Kollektiven abgrenzt (vgl. Gardt 2003: 271, Vierhaus 1995: 8, 13, Linke 2003: 43).

Wesenhaft für den Wandel sprachlicher Traditionen ist, dass die Schaffung von Neuem zugleich die Fortführung einer Tradition ist, so dass die Sprache es den Sprechern erlaubt „die Tradition zu überwinden, während sie sie fortführen“" (Coseriu 1958/1974: 185). Den Sprachwandel erforschen bedeutet daher zu zeigen, wie die Ausdrucksfreiheit der Sprecher sich in die Rahmungen der Tra- 
dition einfügt. Sprachwandel beginnt also mit einer punktuellen Innovation, die innerhalb des Systems der Sprache durch den schöpferischen Akt eines Individuums hervorgebracht wird. Diese kreativen Akte erlauben es, neue Gedanken auch in neuer Weise zu versprachlichen, sodass das Sprechen als Tätigkeit sich nicht in der Anpassung bereits bestehender Sprachgewohnheiten erschöpft (Coseriu 1958/1974: 92). Coseriu nimmt damit den bereits zitierten Gedanken Menéndez Pidals auf, dass Tradition und Sprachstruktur ineinander greifen, wobei die Tradition der Struktur vorausgeht (1954: 187). Coseriu stimmt dabei dem von Menéndez Pidal formulierten Primat des Historischen vor dem Systematischen zu, kritisiert jedoch die implizit vorausgesetzte Opposition von System und Tradition und radikalisiert diese Idee:

Die Sprache ist nicht zuerst System und dann Tradition oder umgekehrt, sondern sie ist gleichzeitig und in jedem Augenblick „systematische Tradition“ und „traditionelles System“. (Coseriu 1958/1974: 184)

Da die Sprache ein "traditionelles System“ ist, liefert die auf die kreative Neuschöpfung folgende Traditionswerdung des Neuen den Schlüssel zum Verständnis der dynamischen Geschichtlichkeit von Sprache. Den Kern des linguistischen Interesses bildet daher die graduelle Entwicklung des Neuen zu einer „systematischen Tradition“. Diese Fokussetzung auf der Traditionswerdung impliziert, dass Sprachwandel weniger als eine vom Existierenden abweichende Veränderung gesehen wird als vielmehr in einer perspektivischen Umkehrung als Festigung von Traditionen (Coseriu 1958/1974: 91).

Der von Coseriu für den Sprachwandel formulierte Grundriss ist auch für den Wandel der Diskurstraditionen grosso modo gültig. So wie sprachliche Neuschöpfungen auf individuelle Sprechakte zurückgehen, so sind auch Innovationen im diskurstraditionellen Wissen auf die Kreativität einzelner Individuen zurückzuführen und breiten sich dann in kulturellen Gruppierungen oder über diese hinweg durch Übernahme aus. Gemeinsam ist sprachlichen Traditionen und Diskurstraditionen, dass die Traditionswerdung von Innovationen mit einem Prozess der Einpassung in das bestehende Traditionsgefüge einhergeht. Dabei handelt es sich im Fall der Diskurstraditionen jedoch nicht um ein analog zum Sprachsystem gebautes Gefüge. Vielmehr werden neue Diskurstraditionen in die traditionellen Ensembles der Diskursuniversen und damit in den „kommunikativen Haushalt“ nach Luckmann $(1988,1997)$ integriert.

Auch die Erkenntnis, dass der Sprachwandel erst in seiner Zweiheit als Neuerung und Fortführung von Traditionen wirklich verstehbar ist, gilt analog für die Diskurstraditionen. Auf der Ebene des diskurstraditionellen Wissens erschließt sich der Wandel erst wirklich, wenn man ihn nicht einseitig als Änderung, sondern in einer Umkehrung der Perspektive als Festigung und Fortschreibung von Traditionen sieht: Das Traditionelle ist so gesehen dann ein 
Gleichgewicht gegen die vielfältigen Variationen, denen das Sprechen in den verschiedenen Kommunikationssituationen unterliegt. Im Fall des Sprachwandels liegt nach Coseriu das zentrale Interesse nicht bei der Entstehung der Innovation, sondern vielmehr in deren Traditionswerdung. Beim Vergleich beider Traditionalitäten stellt sich die Frage, ob diese Schwerpunktsetzung für die Diskurstraditionen analog gilt oder ob beim diskurstraditionellen Wissen die individuelle Neuschöpfung nicht einen anderen Status hat und daher gegenüber der Traditionswerdung eine Aufwertung erfahren und verstärkt in den Fokus der kulturorientierten Linguistik rücken sollte.

Klärend für das Wesen der sprachlichen Tradition(en) ist ferner die Differenzierung von System und Norm. Während das System die Möglichkeiten eines verständlichen Sprechens umfasst, bildet die Norm die Summe der in einer Sprache üblichen sprachlichen Realisierungen.

Das System umfasst die idealen Realisierungsformen einer Sprache, das heißt, die Technik und die Regeln des entspechenden Sprachschaffens; die Norm, die mit dieser Technik und nach jenen Regeln bereits realisierten Muster. Auf diese Weise stellt das System die Dynamik der Sprache dar, die Art und Weise ihres Werdens, und folglich ihre Möglichkeit, über das bereits realisierte hinauszugehen; die Norm dagegen entspricht der Fixierung der Sprache in traditionellen Mustern [...]. (Coseriu 1958/1974: 47f., Hervorhebungen im Original)

Den Realisierungsformen des dynamischen Systems steht damit die Norm als Summe bereits realisierter traditioneller Muster gegenüber. Coseriu erfasst diese Unterscheidung über die Realisierung und stellt den „idealen Realisierungsformen“ die „bereits realisierten Muster" gegenüber. Man kann diese Differenzierung aber auch stärker auf die Tradition hin ausrichten, denn aus Coserius Definition wird klar, dass das Konzept der Tradition für System und Norm eine unterschiedliche Rolle spielt. So sind die Möglichkeiten des Systems Wege des Sprechens, die zu Traditionen werden können, und damit potentielle Traditionen. Dagegen sind die üblichen Realisierungen der Norm bereits existierende und damit aktuelle Traditionen: Die Traditionen der Norm sind dadurch definiert, dass sie bereits realisiert und überliefert wurden.

Das Phänomen systematischer Schöpfungen, die gegen die Norm verstoßen (ich nehmte statt ich nahm), führt zu einem weiteren Merkmal sprachlicher Traditionen. Nach Coseriu haben im System angelegte Normverstöße „in Zeiten schwacher Tradition und kulturellen Verfalls“ (1958/1974: 117) gute Möglichkeiten sich auszubreiten. Die rasche und leichte Integration normwidriger Neuschöpfungen in das traditionelle Wissen wird von Coseriu als Schwäche der Traditionen ausgelegt, wogegen systematisch mögliche aber normwidrige Neuschöpfungen an starken Traditionen gewissermaßen abprallen. Versteht man den bei Coseriu wertend formulierten „kulturellen Verfall“ als Neuorientierung kultureller Hegemonien und damit als eine Phase des Übergangs zwischen 
Zentren der (Sprach-)Kultur, dann bleibt der Gedanke einer Unterscheidung zwischen „schwachen“ und „starken“ Traditionen. Da es sich bei der Stärke und Schwäche der Traditionen um ein Kontinuum handelt, könnte man hier neutraler formuliert - von einem mehr oder weniger großen Spielraum sprechen, den es innerhalb einer Tradition für Variationen gibt oder aber von einer mehr oder weniger strengen Fixierung der Traditionen.

Geht man vom sprachlichen System als Gefüge von Strukturen und Paradigmen aus, dann wird schnell klar, dass die Diskurtraditionen nicht in der gleichen Weise „systematisch“ sind. Im Fall der Diskurstraditionen gibt es aufgrund der nicht vorhandenen Systemhaftigkeit kein vergleichbares „System der Möglichkeiten“, sondern lediglich die „Norm“ im Sinne der traditionellen, bereits realisierten kulturellen Muster des Sprechens. Diese Differenz zeigt sich auch darin, dass Diskurstraditionen zwar oft Teil einer Textsorte oder Gattung sind, jedoch auch als autonome Traditionen "für sich" funktionieren können, wie dies etwa bei Grußformeln als Traditionen der Begrüßung und Gesprächseröffnung der Fall ist. Selbst Diskurstraditionen, die eng in eine fest definierte Gattung wie etwa das Sonett integriert sind, haben niemals den gleichen Grad an systematischer Integration wie eine idiomatische Tradition.

Diese Absenz eines der langue homologen Systems bedeutet allerdings nicht, dass nicht auch die Diskurstraditonen größere, in sich organisierte Einheiten bilden. So sind Diskurstraditionen Elemente des „kommunikativen Haushalts“ (vgl. Luckmann 1988: 282; 1997: 12-14) einer Kulturgemeinschaft und bilden in diesem Haushalt strukturierte Ensembles, die verschiedenen Diskursuniversen angehören und durch diese Zugehörigkeiten eine Ordnung erfahren. Dennoch hat dieses diskurstraditionelle Gefüge keine Systemhaftigkeit im Sinne der langue. Diesen Unterschied verdeutlicht ein Blick auf die mit den Ebenen des Sprachlichen verbundenen Sprecherurteile (Coseriu 1988: 88-89). Dabei ist mit der einzelsprachlichen Ebene das Urteil der Korrektheit verbunden, während auf der individuellen Ebene Äußerungen nach dem Kriterium der Angemessenheit beurteilt werden. So erfordert die Beherrschung einer Sprache, dass ein Sprecher nicht nur einzelne idiomatische Traditionen beherrscht, sondern dass er ein systemhaft verbundenes idiomatisches Wissen hat, das es ihm erst erlaubt, das mit den Einzelsprachen verbundene Kriterium der Korrektheit (Coseriu 1988: 89) zu erfüllen. Zwar trägt auch die Beherrschung möglichst vieler Diskurstraditionen eines Diskursuniversums dazu bei, dass das Kriterium angemessenen Sprechens gut erfüllt werden kann, doch können auch vereinzelt beherrschte Diskurstraditionen diese Angemessenheit sichern. Dazu ein Beispiel: Ein deutscher Geschäftsmann, der mit argentinischen Partnern auf Spanisch verhandeln will, muss die idiomatischen Traditionen des Spanischen systemhaft beherrschen, um in einer bestimmten Situation erfolgreich zu agieren, er muss jedoch nicht sämtliche Diskurstraditionen der geschäftlichen 
Dialogführung im argentinischen Kulturraum beherrschen, um in einer konkreten Situation angemessen zu sprechen. Während das idiomatische Wissen daher ein Sprechenkönnen gemäß der Tradition einer Gemeinschaft ist, sind die Diskurstraditionen gewissermaßen ein Sprechenkönnen gemäß den Traditionen, die eine bestimmte Kommunikationssituation verlangt.

Auch wenn die Differenzierung von System und Norm nicht auf das diskurstraditionelle Wissen übertragbar ist, charakterisiert das Kriterium der unterschiedlich starken Fixierung beide Traditionalitäten. Auch Diskurstraditionen können in unterschiedlichem Grade fixiert sein und bieten unterschiedlich große Spielräume, was auch mit den Diskursuniversen, denen sie angehören, zusammenhängt. So sind etwa Diskurstraditionen in der Domäne des Rechts oder der Religion weit stärker fixiert als im Diskursuniversum der Literatur oder des sprachlichen Alltags. Damit gilt für beide Traditionalitäten: Je weniger streng eine Tradition fixiert ist, umso mehr Spielraum beinhaltet sie und umso leichter und häufiger werden Schöpfungen, die gegen die sprachliche oder diskurstraditionelle Norm verstoßen, Teil des traditionellen Wissens.

\subsection{Tradition und Identität}

Sowohl das einzelsprachliche als auch das diskurstraditionelle Wissen sind als Wissensbestand historisch, traditionell, sozial und kollektiv. Der soziale Charakter dieses Wissens impliziert, dass es aktuell in der Sprachgemeinschaft gebraucht wird (Coseriu 1958/1974: 52). In dieser Gemeinschaft werden Traditionen des Sprechens von den Individuen „als Glieder historischer Gemeinschaften" ausgeübt (1958/1974: 38). Die Traditionen schaffen die Gemeinschaft und sie halten die Gemeinschaft zusammen (vgl. auch Garatea Grau 2005: 76). Das Sprechen in Traditionen impliziert, dass Sprecher grundsätzlich bereit sind, die Sprache der Sprachgemeinschaft, der sie angehören, zu benutzen, da sie diese sprachlichen Gewohnheiten als die ihrigen begreifen und annehmen. Diese Bereitschaft resultiert aus der Geschichtlichkeit des Menschen, der die sprachlichen Traditionen als Teil seiner Identität ansieht (Coseriu 1958/1974: 59). Das „Sprechenkönnen gemäß der Tradition einer Gemeinschaft“ zeigt sich positiv darin, dass der Sprecher die Traditionen seiner Sprache kennt und benutzt, und es zeigt sich ex negativo darin, dass der Sprecher Formen, die nicht der Tradition seines Sprechenkönnens angehören, als fremd betrachtet (1958/1974: 38, 50). Ferner ist die Traditionalität des Sprechens durch das Universale der Alterität bedingt. Denn wenn ein Sprecher um verstanden zu werden spricht wie andere, dann hat diese Alterität immer eine historische Dimension: Das Sprechen wie andere impliziert, dass man so spricht, wie auch zu früheren Zeitpunkten bereits gesprochen wurde (1958/1974: 60). 
Ein Pfeiler der identitätsbildenden Kraft sprachlicher Traditionen liegt nach Coseriu in der Besonderheit, dass die Sprache diachronisch betrachtet ein "Gefüge von traditionellen sprachlichen Modi“ (1958/1974: 41) ist, die in der Sprachgemeinschaft überliefert werden, während sie synchronisch ein Gefüge von gemeinsamen und „aktuellen“ Ausdrucksweisen ist. Traditionalität und Aktualität bilden dabei keinen Gegensatz, da die aktuelle Gemeinsamkeit gerade auf der Überlieferung der sprachlichen Ausdrucksweisen beruht. In dieser Perspektive wirken Traditionen in zweifacher Weise gemeinschaftsbildend: erstens durch die Überlieferung, die die Sprecher zu einer Gemeinschaft der tradierenden Akteure macht, und zweitens durch die gemeinschaftlich erlebte Aktualität der Sprachgewohnheiten in der sprachlichen Verständigung. Daher stiften sprachliche Traditionen Gemeinschaft sowohl durch die in der Tradition gespeicherte Überlieferung als auch durch die in ihr enthaltene Aktualität.

Das Sprechen in Diskurstraditionen kann ebenfalls als allgemeine anthropologische Größe betrachtet werden: Der Sprecher benutzt die Diskurstraditionen der kulturellen Gruppierungen, denen er angehört, und erkennt die Diskurstraditionen anderer kultureller Gruppierungen als fremd. Grundsätzlich sind die Verbünde, die Diskurstraditionen tragen und ausüben - anders als durch die sprachliche Tradition konstituierte Sprachgemeinschaften -, lockere soziale Konfigurationen, die sich in vielfältiger Weise überlagern und nur im Ausnahmefall ein klar abgrenzbares Kollektiv bilden, dessen Geschichte man erzählen kann. Aus dieser Verfasstheit der sozialen und kulturellen Konfigurationen kontrastiv zur Sprachgemeinschaft folgt eine weitere Besonderheit der Diskurstraditionen, die sie von den idiomatischen Traditionen unterscheidet: ihre potentielle Diskontinuität. So können Diskurstraditionen, die über längere Zeit nicht mehr ausgeübt wurden, wieder reaktiviert werden und haben in diesem Sinne eine potentiell transhistorische Traditionalität. Ein Beispiel hierfür sind antike Diskursmuster, die in der Renaissance wieder aktualisiert und imitiert werden (vgl. Cremer in diesem Band). Aus dieser transhistorischen Verfasstheit der Diskurstraditionen folgt nun eine weitere Besonderheit diskurstraditioneller Gruppierungen. Während Sprachgemeinschaften sich durch die Kontinuität idiomatischer Traditionalität stets als durchgängig existierende Gemeinschaft manifestieren, können die kulturellen Gruppierungen, die Diskurstraditionen tragen und vermitteln, sich diskontinuierlich über zeitliche Unterbrechungen hinweg konstituieren. So können etwa Gelehrte und Dichter der Antike und deren "Wiederentdecker" in der Renaissance als eine kulturelle Gruppierung aufgefasst werden, die über Zeit und Raum hinweg kulturell-literarische Traditionen der Textgestaltung praktiziert. Doch obwohl die Diskurstraditionen nicht analog zu den Sprachen Gemeinschaften bilden, sondern auf bestehenden Kulturgemeinschaften aufbauen, haben sie die Wirkung, kulturelle Gruppierungen zu stärken. Denn wie die Sprachgewohnheiten beinhalten die Diskurs- 
traditionen als gegenwärtig praktizierte Techniken zugleich die Werte der Überlieferung und der Aktualität. Die Sprecher erfahren die Diskurstraditonen zugleich als ein von ihnen gemeinsam überliefertes Wissen und sie erleben das aktuelle Funktionieren der Diskurstraditionen in der Kommunikation. Beides stärkt die Zusammengehörigkeit der kulturellen Konfiguration und verleiht ihr so Dauer.

\subsection{Tradition und Muster}

Die Aufarbeitung des Traditionsbegriffes innerhalb des tradicionalismo pidaliano und des Coseriu'schen Systems ist eine Möglichkeit, den Begriff der Tradition zu schärfen. Eine weitere Möglichkeit besteht darin, Tradition und Traditionalität mit verwandten Begriffen abzugleichen. Dies soll hier mit dem Begriff des Musters, den bereits Heringer (1974: 20f., 38ff.) in der Germanistik, und zwar im Anschluss an Wittgenstein, prominent gemacht hat, skizzenhaft geschehen. Ein grundliegendes Anliegen der kulturbezogenen Sprachwissenschaft ist nach Gardt die Herausarbeitung des Musterhaften in den verschiedenen Sprachverwendungen (vgl. Gardt 2012a: 299). Muster werden dabei als transphrastische Formungen von Texten und Textteilen verstanden, die über den einzelnen Text hinaus die sprachliche Gestaltung von Texten als Realisierung von Typen charakterisieren und damit überindividuellen Charakter haben (Gardt 2007: 31f. und 2012a: 299). Die Konzentration auf das Musterhafte der sprachlichen Gestaltung wird dabei als ein Schwerpunkt gesehen, der der Diskursanalyse die Rückbindung an die Sprache und damit die „linguistische Identität" sichert (Gardt 2007: 42, 43). In den Überlegungen Gardts liegt der Fokus damit auf der Rolle des Musters für die Diskurslinguistik und meint Textmuster, die sich im (virtuellen) Korpus eines Diskurses rekurrent manifestieren.

Vergleicht man die Konzepte Muster und Tradition, dann stellen beide interindividuelle und über den einzelnen Sprechakt hinausgehende Größen dar, die wiederholt auftreten bzw. angewendet werden. Der Unterschied ist, dass eine Tradition stets historisch ist, wogegen ein Muster sowohl historisch als auch universell sein kann: Muster können sich verändern, doch impliziert der Begriff nicht notwendig eine wesenhafte Veränderung in der Zeit. Daher können bspw. sowohl das universelle turn-taking als auch die stark historischkulturell geprägten Techniken der Eröffnung und Beendigung eines Privatbriefs als Muster der Textgestaltung aufgefasst werden. Ein weiterer Unterschied zwischen beiden Begriffen wird deutlich, wenn man bedenkt, dass Muster etwas sind, das man in Texten sucht und erkennt - nicht zufällig spricht man von „Mustererkennung“. Während Traditionen also etwas sind, das man anwendet, 
sind Muster etwas, das man in Texten und Diskursen aufspürt und identifiziert: Die Muster sind die Spuren der Ausübung einer Tradition.

Zwei Schlussfolgerungen lassen sich (vorläufig) daraus ziehen. Zum einen ist die Tradition das dem Muster übergeordnete Konzept, denn die Tradition besteht aus Mustern, die man anwendet und die der Gesprächspartner wiedererkennt und einer Tradition der Textgestaltung zuordnet. Zum anderen scheint die (implizite) Differenzierung von Muster und Tradition in der Forschung mit der Unterscheidung von dynamis und ergon einherzugehen: Während die Tradition zur dynamis gehört, erscheint das Muster als eine im Text als ergon manifestierte Größe, die der Linguist in den Texten und Diskursen erkennt und durch linguistisches Interpretieren extrahiert. Während die Tradition vom Sprecher ausgeübt wird, entsteht das Muster gleichsam im Auge des Interpreten. Die hier nur angedeutete Überblendung der bisher unverbundenen Begriffe von Muster und Tradition (à suivre) macht deutlich, dass auch die vergleichende Analyse von Konzepten des Überindividuellen dazu beitragen kann, die doppelte Traditionalität von Texten und Diskursen zu erhellen.

\section{Schlussbetrachtung}

Die romanistisch geprägte Theorie der Diskurs- und Texttraditionen, die sich aus Coserius „Linguistik des Sprechens“ entwickelt hat, wählt als Ausgangspunkt die parole und damit das induktive und semasiologische Verfahren einer bottom up-Analyse. Ganz nah bei den Dingen, rekonstruiert sie die in der parole sich manifestierende Aktivität des Diskurses und sein Produkt, den Text, in seiner Zugehörigkeit zu mehr oder weniger flexiblen und dabei in unterschiedlicher Dynamik sich verändernden Traditionen, die ihrerseits bestimmten Diskursuniversen zuordenbar sind. Insofern wir noch weit davon entfernt sind, auch nur annähernd einen Überblick über die vielfältigen Berührungspunkte und Verflechtungen zwischen den verschiedenen Diskurs- und Texttraditionen zu haben, sind die Grenzen dieses Forschungsansatzes noch längst nicht ausgelotet; sie dürften jedoch dort liegen, wo Zusammenhänge zwischen Traditionen nicht mehr signifikant wahrnehmbar sind oder möglicherweise auch überhaupt nicht existieren.

Ausgehend von Foucaults Diskursbegriff hat sich zunächst in Frankreich und dann in Deutschland, hierzulande jedoch wesentlich stärker in der Germanistik als in der Romanistik, parallel eine ganz andere Forschungsrichtung entwickelt, die sich inzwischen nicht mehr, wie noch in Frankreich, Diskursanalyse (analyse $d u$ discours), sondern Diskurslinguistik - und zwar, mit einem Wortspiel, „Diskurslinguistik nach Foucault" - nennt (Warnke 2007) und neuerdings auch als eine „angewandte Diskurslinguistik“ disziplinär etablieren 
möchte (Roth/Spiegel 2013). Diese Diskurslinguistik signalisiert Anschlussfähigkeit an die vielen Spielarten der Diskursanalyse, wie sie sich z. B. in der Geschichts-, Literatur- und Sozialwissenschaft bereits länger etabliert haben (Wrana u. a. 2014; vgl. dazu Lebsanft 2015). Ihr Ausgangspunkt ist, als ein für linguistische Zwecke „zurechtgemachtes“ oder kritisch angeeignetes Verständnis von Foucault, der Diskurs als ein - wie bei Bußmann $\left({ }^{3} 2002,{ }^{4} 2008\right)$ bereits in der eingangs zitierten Weise terminologisch fixiert - im weitesten Sinn inhaltlich oder thematisch, also onomasiologisch konzipiertes und abgegrenztes Textkorpus, das sich „transtextuell“, in ganz unterschiedlichen Texttypen und Textsorten manifestieren kann (Busse/Teubert 1994: 14; vgl. Spitzmüller/ Warnke 2011: 82). Im Mittelpunkt dieses Diskursbegriffs steht „das handlungsbegleitende und sozial stratifizierende kollektive Wissen bestimmter Kulturen und Kollektive" (Spitzmüller/Warnke 2011: 8). Die Diskurslinguistik dieser Prägung verspricht also gewissermaßen, nach dem deduktiven Verfahren der top down-Analyse die Übergänge zwischen den durch Wissenssysteme bestimmten „Dingen“ und „Wörtern“ (Foucault 1966) zu füllen und dabei auch Foucaults „letzte“, dort aus der Betrachtung ausgeschlossene "Stufe“ des materiellen Textes einzubeziehen. Das entsprechende Forschungsprogramm verfügt inzwischen über beeindruckend ausgearbeitete heuristische Instrumente, die systematisch die verschiedenen Ebenen einer diskurslinguistischen Analyse erfassen. $\mathrm{Zu}$ nennen sind hier etwa das von Gardt seit 2002 erarbeitete textsemantische Analyseraster (TexSem) zur Bedeutungserschließung in Text und Diskurs (vgl. Gardt 2002: 228-129, 2008: 207-210, 2012b: 64-67 und 2013: 4850) und die diesem Schema verwandte „Diskurslinguistische Mehr-EbenenAnalyse“ (DIMEAN) von Warnke/Spitzmüller (2008: 23-45; in revidierter Form in Spitzmüller/Warnke 2011: 197-201). Im „Layout“ der zuletzt genannten Methode bilden die "Akteure“ das Scharnier zwischen „intratextuell“ analysierten Einzeltexten und übergreifend untersuchten "transtextuellen“ Strukturen, die kulturelle und gesellschaftliche Wissensbestände aufgreifen (Spitzmüller/ Warnke 2011: 136, 201).

Bei näherer Betrachtung zeigt sich, dass die intratextuelle Analyse der Diskurslinguistik sich ganz klassischer, geradezu rigid strukturalistisch geprägter textlinguistischer Methoden bedient. Es ist nicht undenkbar, dass an beiden Punkten die Einsichten der Romanistik in die Flexibilität und Dynamik von Diskurs- und Texttraditionen und deren Einbettung in außersprachliche Umfelder ein Feld fruchtbaren interdisziplinären, kulturlinguistischen Austauschs vertiefen könnten. Umgekehrt kann auch die Diskurstraditionenforschung von der Methodologie der Diskursanalyse profitieren. So ist beiden Disziplinen gemeinsam, dass sie Muster und Techniken der Diskursgestaltung an der Schnittstelle von Sprache und Wissen rückgebunden an kulturelle Gruppierungen untersuchen. Ein Unterschied beider Herangehensweisen besteht darin, dass 
die Diskursanalyse einen ausgeprägteren Fokus auf sprachliche Aushandlungen setzt, da sie den Diskurs immer als Repräsentation und Movens einer sprachlichen Interaktion zu gesellschaftlich relevanten Themen auffasst (vgl. Gardt 2007). Die Diskursanalyse akzentuiert damit den Gesichtspunkt der Tätigkeit (energeia) stärker als die auf die dynamis und das Wechselspiel der Wissensbestände konzentrierte Erforschung diskurstraditionellen Wissens. Das Desiderat einer Ausweitung der Diskurstraditionenforschung auf die Akteure und deren Handeln kann daher von den Methoden der auf Aushandlungsprozesse spezialisierten Diskursanalyse bzw. Diskurslinguistik profitieren. In dieser Sichtweise stehen Diskurstraditionenforschung und Diskursanalyse in einer Relation spannungsreich-produktiver Verwandtschaft innerhalb der kulturorientierten Linguistik.

\section{Bibliographie}

Albrecht, Jörn (2003): Können Diskurstraditionen auf dem Wege der Übersetzung Sprachwandel auslösen? - In: Heidi Aschenberg, Raymund Wilhelm (Hg.): Romanische Sprachgeschichte und Diskurstraditionen. Akten der gleichnamigen Sektion des XXVII. Deutschen Romanistentages, 37-53. - Tübingen: Narr.

Aschenberg, Heidi/Wilhelm, Raymund (Hg.) (2003): Romanische Sprachgeschichte und Diskurstraditionen. Akten der gleichnamigen Sektion des XXVII. Deutschen Romanistentages. - Tübingen: Narr.

Assmann, Aleida ( $\left.{ }^{5} 1997\right)$ : Gedächtnis, Erinnerung. - In: Klaus Bergmann u. a. (Hg.): Handbuch der Geschichtsdidaktik, 33-37. Seelze-Velber: Kallmeyer.

Bally, Charles (1932/ $\left.{ }^{4} 1965\right)$ : Linguistique générale et linguistique française. - Paris: Leroux, Bern: Francke.

Barthes, Roland (1966): Introduction à l'analyse structurale des récits. - In: Communications 8, 1-27.

Benveniste, Émile (1954/1976): Tendances récentes en linguistique générale. - In: ders.: Problèmes de linguistique générale I, 3-17. Paris: Gallimard (zuerst in: Journal de psychologie normale et pathologique, 47-51, 130-145).

Benveniste, Émile (1956a/1976): La nature des pronoms. - In: ders.: Problèmes de linguistique générale I, 251-257. Paris: Gallimard (zuerst in: Morris Halle u. a. [Hg.]: For Roman Jakobson. Essays on the Occasion of his Sixtieth Birthday, 11 October 1956, 34-37. Den Haag: Mouton).

Benveniste, Émile (1956b/1976): Remarques sur la fonction du langage dans la découverte freudienne. - In: ders.: Problèmes de linguistique générale I, 75-87. Paris: Gallimard (zuerst in: La Psychanalyse 1, 3-16).

Benveniste, Émile (1959/1976): Les relations de temps dans le verbe français. - In: ders.: Problèmes de linguistique générale I, 237-250. Paris: Gallimard (zuerst in: Bulletin de la Société Linguistique de Paris 54, 69-82).

Benveniste, Émile (1970/1981): L'appareil formel de l'énonciation. - In: ders.: Problèmes de linguistique générale II, 79-88. Paris: Gallimard (zuerst in: Langages 17, 12-18). 
Brinker, Klaus u. a. (Hg.) (2000-2001): Text- und Gesprächslinguistik. Ein internationales Handbuch zeitgenössischer Forschung, 2 Bde. - Berlin/New York: De Gruyter.

Busse, Dietrich/Teubert, Wolfgang (1994): Ist Diskurs ein sprachwissenschaftliches Objekt? Zur Methodenfrage der historischen Semantik. - In: Dietrich Busse, Fritz Hermanns, Wolfgang Teubert (Hg.): Begriffsgeschichte und Diskursgeschichte. Methodenfragen und Forschungsergebnisse der historischen Semantik, 10-28. Opladen: Westdeutscher Verlag.

Bußmann, Hadumod (1983, $\left.{ }^{2} 1990,{ }^{3} 2002,{ }^{4} 2008\right)$ : Lexikon der Sprachwissenschaft. - Stuttgart: Kröner.

Coseriu, Eugenio (1955-1956): Determinación y entorno. Dos problemas de una lingüística del hablar. - In: Romanistisches Jahrbuch 7, 29-54.

Coseriu, Eugenio (1975): Determinierung und Umfeld. Zwei Probleme einer Linguistik des Sprechens. - In: ders.: Sprachtheorie und allgemeine Sprachwissenschaft. 5 Studien, 253290. München: Fink.

Coseriu, Eugenio (1958/1974): Synchronie, Diachronie und Geschichte. - München, Wilhelm Fink Verlag.

Coseriu, Eugenio (1980/31994): Textlinguistik. Eine Einführung. - Tübingen: Narr, Tübingen/Basel: Francke.

Coseriu, Eugenio (1988): Sprachkompetenz. Grundzüge der Theorie des Sprechens. - Tübingen: Francke.

Cremer, Désirée (2015): Die Consolatio-Übersetzung von Colard Mansion: Zum Auftakt der französischen Elegie und weiterer texttraditioneller Phänomene. - In: Franz Lebsanft, Angela Schrott (Hg.): Diskurse, Texte, Traditionen. Modelle und Fachkulturen in der Diskussion. Göttingen: Vandenhoeck \& Ruprecht/Bonn University Press [in diesem Band].

Ehlich, Konrad (Hg.) (1994): Diskursanalyse in Europa. - Frankfurt a. M. u. a.: Lang.

Fohrmann, Jürgen (1997): Diskurs. - In: Klaus Weimar u. a. (Hg.): Reallexikon der deutschen Literaturwissenschaft. Neubearbeitung des Reallexikons der deutschen Literaturgeschichte, 369-372. Berlin/New York: De Gruyter.

Foucault, Michel (1966): Les mots et les choses. Une archéologie des sciences humaines. - Paris: Gallimard.

Foucault, Michel (1969): L'archéologie du savoir. - Paris: Gallimard.

Foucault, Michel (1971): L'ordre du discours. Leçon inaugurale au Collège de France prononcée le 2 décembre 1970. - Paris: Gallimard.

Garatea Grau, Carlos (2005): El problema del cambio lingüístico en Ramón Menéndez Pidal. El individuo, las tradiciones y la historia. - Tübingen: Narr.

Gardt, Andreas (2002): Wort, Text und Bedeutung. Aspekte der semantischen Erschließung von Texten. - In: Vilmos Ágel, Andreas Gardt, Ulrika Haß-Zumkehr, Thorsten Roelcke (Hg): Das Wort. Seine strukturelle und kulturelle Dimension. Festschrift für Oskar Reichmann zum 65. Geburtstag, 111-132. Berlin/New York: De Gruyter.

Gardt, Andreas (2003): Sprachwissenschaft als Kulturwissenschaft. - In: Ulrike HaßZumkehr, Christoph König (Hg.): Literaturwissenschaft und Linguistik von 1960 bis heute, 271-288. Göttingen: Wallstein Verlag.

Gardt, Andreas (2007): Diskursanalyse. Aktueller theoretischer Ort und methodische Möglichkeiten. - In: Ingo Warnke (Hg.): Diskurslinguistik nach Foucault. Theorie und Gegenstände, 28-52. Berlin/New York: De Gruyter. 
Gardt, Andreas (2008): Kunst und Sprache. Beobachtungen anlässlich der documenta 12. In: Achim Barsch, Helmut Scheuer, Georg-Michael Schulz (Hg.): Literatur - Kunst - Medien. Festschrift für Peter Seibert zum 60. Geburtstag, 201-224. München: Meidenbauer.

Gardt Andreas (2012a): Sprachgeschichte als Kulturgeschichte. Chancen und Risiken der Forschung. - In: Péter Maitz (Hg.): Historische Sprachwissenschaft. Erkenntnisinteressen, Grundlagenprobleme, Desiderate, 289-300. Berlin/Boston: De Gruyter.

Gardt, Andreas (2012b): Textsemantik. Methoden der Bedeutungserschließung. - In: Jochen A. Bär, Marcus Müller (Hg.): Geschichte der Sprache und Sprache der Geschichte. Probleme und Perspektiven der historischen Sprachwissenschaft des Deutschen. Oskar Reichmann zum 75. Geburtstag, 61-82. Berlin: Akademie-Verlag.

Gardt, Andreas (2013): Textanalyse als Basis der Diskursanalyse. Theorie und Methoden. In: Ekkehard Felder (Hg.): Faktizitätsherstellung in Diskursen. Die Macht des Deklarativen, 29-55. Berlin/Boston: De Gruyter.

Goffman, Erving (1971): Interaktionsrituale. Über Verhalten in direkter Kommunikation. Frankfurt a. M.: Suhrkamp.

Harris, Zellig S. (1951): Methods in Structural Linguistics. Chicago: University Press.

Harris, Zellig S. (1952): Discourse Analysis. - In: Language 28, 1-30.

Harris, Zellig S. (1969): Analyse du discours. - In: Langages 13, 8-45.

Harris, Zellig S. (1976): Textanalyse. - In: Elisabeth Bense, Peter Eisenberg, Hartmut Haberland (Hg.): Beschreibungsmethoden des amerikanischen Strukturalismus, 261-298. München: Hueber.

Hempfer, Klaus W. (1973): Gattungstheorie. Information und Synthese. - München: Fink.

Heringer, Hans Jürgen (1974): Praktische Semantik. - Stuttgart: Klett.

Jacob, Daniel/Kabatek, Johannes (Hg.) (2001): Lengua medieval y tradiciones discursivas en la Península Ibérica: descripción gramatical - pragmática histórica - metodología. - Frankfurt a. M./Madrid: Vervuert/Iberoamericana.

Kabatek, Johannes (Hg.) (2008): Sintaxis histórica del español y cambio lingüístico: Nuevas perspectivas desde las Tradiciones Discursivas. - Frankfurt a. M./Madrid: Vervuert/Iberoamericana.

Kabatek, Johannes (2015): Warum die „Zweite Historizität“ eben doch die zweite ist - von der Bedeutung von Diskurstraditionen für die Sprachbetrachtung. - In: Franz Lebsanft, Angela Schrott (Hg.): Diskurse, Texte, Traditionen. Modelle und Fachkulturen in der Diskussion. Göttingen: Vandenhoeck \& Ruprecht/Bonn University Press [in diesem Band].

Koch, Peter (1997): Diskurstraditionen: zu ihrem sprachtheoretischen Status und ihrer Dynamik. - In: Barbara Frank, Thomas Haye, Doris Tophinke (Hg.): Gattungen mittelalterlicher Schriftlichkeit, 43-79. Tübingen: Narr.

Koch, Peter (2008): Tradiciones discursivas y cambio lingüístico: El ejemplo del tratamiento vuestra merced en español. - In: Johannes Kabatek (Hg.): Sintaxis histórica del español y cambio lingüístico: Nuevas perspectivas desde las Tradiciones Discursivas, 53-87. Frankfurt a. M./Madrid: Vervuert/Iberoamericana.

Koch, Peter/Oesterreicher, Wulf ('2011): Gesprochene Sprache in der Romania. Französisch, Italienisch, Spanisch. - Berlin/New York: De Gruyter.

Lebsanft, Franz (2005): Kommunikationsprinzipien, Texttraditionen, Geschichte. - In: Angela Schrott, Harald Völker (Hg.): Historische Pragmatik und historische Varietätenlinguistik in den romanischen Sprachen, 25-43. Göttingen: Universitätsverlag Göttingen. 
Lebsanft, Franz (2015): Rezension von Wrana u. a. (Hg.) 2015. - In: Romanische Forschungen 127, 291-293.

Linke, Angelika (2003): Sprachgeschichte - Gesellschaftsgeschichte - Kulturanalyse. - In: Helmut Henne, Horst Sitta, Herbert Ernst Wiegand (Hg.): Germanistische Linguistik: Konturen eines Faches, 25-65. Tübingen: Niemeyer.

Luckmann, Thomas (1988): Kommunikative Gattungen im kommunikativen „Haushalt“ einer Gesellschaft. - In: Gisela Smolka-Koerdt, Peter M. Spangenberg, Dagmar TillmannBartylla (Hg.): Der Ursprung von Literatur. Medien, Rollen, Kommunikationssituationen zwischen 1450 und 1650, 279-288. München: Fink.

Luckmann, Thomas (1997): Allgemeine Überlegungen zu kommunikativen Gattungen. - In: Barbara Frank, Thomas Haye, Doris Tophinke (Hg.): Gattungen mittelalterlicher Schriftlichkeit, 11-17. Tübingen: Narr.

Menéndez Pidal, Ramón (1926/81976): Orígenes del español. Octava edición, según la tercera, muy corregida y adicionada. - Madrid: Espasa-Calpe.

Menéndez Pidal, Ramón (1942/91991): Poesía juglaresca y juglares. Orígenes de las literaturas románicas. - Madrid: Espasa-Calpe.

Menéndez Pidal, Ramon (1945): Unidad de la lengua. - In: ders.: Castilla, la tradición, el idioma, 173-218. Buenos Aires: Espasa-Calpe Argentina.

Menéndez Pidal, Ramón (1952): Miscelánea histórico-literaria. - Buenos Aires: Espasa-Calpe.

Menéndez Pidal, Ramón (1953/1968): Romancero hispánico. Teoría e historia. Band 1 und Band 2. - Madrid: Espasa-Calpe.

Menéndez Pidal, Ramón (1954): A propósito de $l$ y $l l$ latinas. Colonización suditálica en España. - In: Boletín de la Real Academia Espanola 34, 165-216.

Oesterreicher, Wulf (1997): Zur Fundierung von Diskurstraditionen. - In: Barbara Frank, Thomas Haye, Doris Tophinke (Hg.): Gattungen mittelalterlicher Schriftlichkeit, 19-41. Tübingen: Narr.

Pagliaro, Antonino (1955): Linguistica della „parola“. - Roma: Ed. dell'Ateneo.

Parr, Rolf (2008): Diskurs. - In: Clemens Kammler, Rolf Parr, Ulrich Johannes Schneider (Hg.): Foucault-Handbuch. Leben - Werk - Wirkung, 233-237. Stuttgart/Weimar: Metzler.

Raible, Wolfgang (1997): Das „Lob der Variante“ aus der Sicht des Sprachwissenschaftlers. In: Martin-Dietrich Gleßgen, Franz Lebsanft (Hg.): Alte und neue Philologie, 127-141. Tübingen: Niemeyer.

Roth, Kersten Sven/Spiegel, Carmen (Hg.) (2013): Angewandte Diskurslinguistik. Felder, Probleme, Perspektiven. - Berlin: Akademie Verlag.

Rüsen, Jörn (1983): Historische Vernunft. Grundzüge einer Historik I: Die Grundlagen der Geschichtswissenschaft. - Göttingen: Vandenhoeck \& Ruprecht.

Saussure, Ferdinand (1916/2013): Cours de linguistique générale. Zweisprachige Ausgabe französisch-deutsch mit Einleitung, Anmerkungen und Kommentar von Peter Wunderli. - Tübingen: Narr.

Schlieben-Lange, Brigitte (1983): Traditionen des Sprechens. Elemente einer pragmatischen Sprachgeschichtsschreibung. - Stuttgart: Kohlhammer.

Schörken, Rolf ( $\left.{ }^{5} 1997\right)$ : Geschichte als Lebenswelt. - In: Klaus Bergmann u. a. (Hg.): Handbuch der Geschichtsdidaktik, 3-9. Seelze-Velber: Kallmeyer.

Schrott, Angela/Völker, Harald (Hg.) (2005): Historische Pragmatik und historische Varietätenlinguistik in den romanischen Sprachen. - Göttingen: Universitätsverlag. 
Spitzmüller, Jürgen/Warnke, Ingo H. (2011): Diskurslinguistik. Eine Einführung in Theorien und Methoden der transtextuellen Sprachanalyse. - Berlin/Boston: De Gruyter.

Szemerényi, Oswald (1982): Richtungen der modernen Sprachwissenschaft. Teil II: Die fünfziger Jahre 1950-1960. - Heidelberg: Winter.

Vierhaus, Rudolf (1995): Die Rekonstruktion historischer Lebenswelten. - In: Hartmut Lehmann (Hg.): Göttinger Gespräche zur Geschichtswissenschaft, Bd. I Wege zu einer neuen Kulturgeschichte, 7-28. Göttingen: Max Planck Institut für Geschichte, Wallstein Verlag.

Warnke, Ingo H. (2007): Diskursanalyse nach Foucault - Dimensionen einer Sprachwissenschaft jenseits textueller Grenzen. - In: Ingo H. Warnke (Hg.): Diskurslinguistik nach Foucault. Theorie und Gegenstände, 3-24. Berlin: De Gruyter.

Warnke, Ingo H./Spitzmüller, Jürgen (Hg.) (2008): Methoden der Diskurslinguistik. Sprachwissenschaftliche Zugänge zur transtextuellen Ebene. - Berlin/New York: De Gruyter.

Wilhelm, Raymund (2001): Diskurstraditionen. - In: Martin Haspelmath, Ekkehard König, Wulf Oesterreicher, Wolfgang Raible (Hg.): Sprachtypologie und sprachliche Universalien. Ein internationales Handbuch. Berlin/New York: De Gruyter, Bd. I, 467-477.

Wrana, Daniel u. a. (Hg.) (2015): DiskursNetz. Wörterbuch der interdisziplinären Diskursforschung. - Berlin: Suhrkamp. 